\title{
Conformations in Solution and in Solid-State Polymorphs: Correlating Experimental and Calculated NMR Chemical Shifts for Tolfenamic Acid
}

Helen Blade ${ }^{a^{*}}$, Charles D. Blundell ${ }^{b^{*}}$, Steven P. Brown ${ }^{c^{*}}$, Jake Carson ${ }^{d}$, Hugh R. W. Dannatt ${ }^{\mathrm{b}}$, Leslie P. Hughes $^{\mathrm{a}^{*}}$ and Anjali K. Menakath ${ }^{\mathrm{c}}$

a Oral Product Development, Pharmaceutical Technology \& Development, Operations, AstraZeneca, Macclesfield, UK

${ }^{\mathrm{b}} \mathrm{C} 4 \mathrm{X}$ Discovery, Manchester, M1 3LD, UK

${ }^{c}$ Department of Physics, University of Warwick, Coventry, CV4 7AL, UK

${ }^{\mathrm{d}}$ Department of Statistics, University of Warwick, Coventry, CV4 7AL, UK

${ }^{*}$ Corresponding authors

E-mail: S.P.Brown@warwick.ac.uk

E-mail: Leslie.Hughes2@astrazeneca.com

Email: Charles.Blundell@c4xdiscovery.com

Email: Helen.Blade@astrazeneca.com 
Supplementary Figure S1 - X-ray powder diffractograms recorded on the material used in this work. A) Form I diffractogram (black) recorded on the material as received from Sigma-Aldrich, compared to that calculated from the Form I crystal structure (orange, CSD accession code 1960856). B) Form II diffractogram (black) recorded on the prepared material (see Materials), compared to that calculated from the Form II crystal structure (magenta, CSD accession code 1960855). The lower comparator is without any preferred orientation, the upper with preferred orientation matching the experimentally observed diffractogram (the prepared material was large, fine needles). Calculations and preparation of the figure was performed using CrystalDiffract (CrystalMaker Software Ltd., UK), using matching line-widths for the calculated diffractograms.
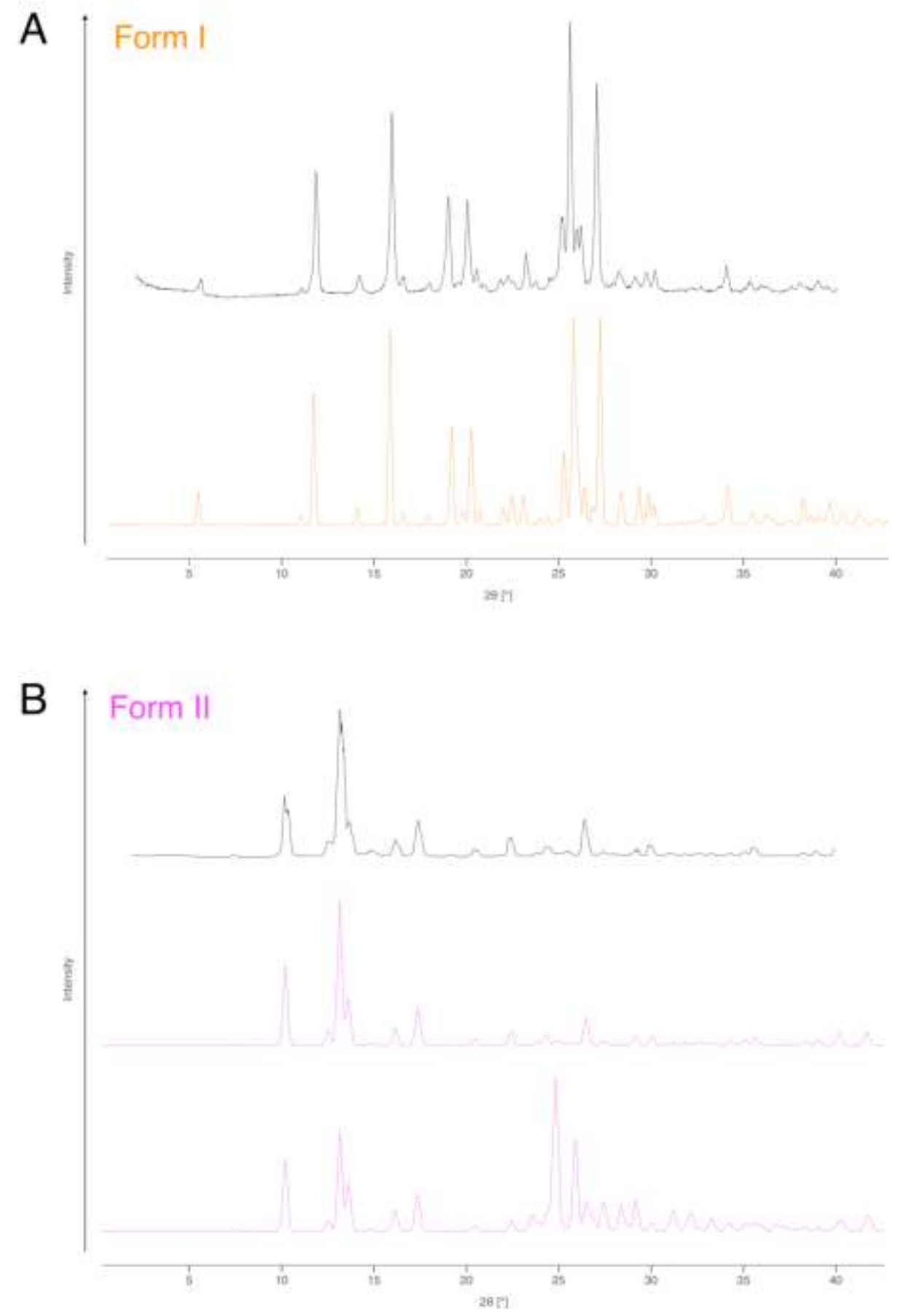
Supplementary Table S1 - Differences in calculated chemical shift (after DFT geometry optimisation) between high resolution (CSD accession codes 1960856 and 1960855) and standard resolution CSD structures (KAXXAI01 and KAXXAI)

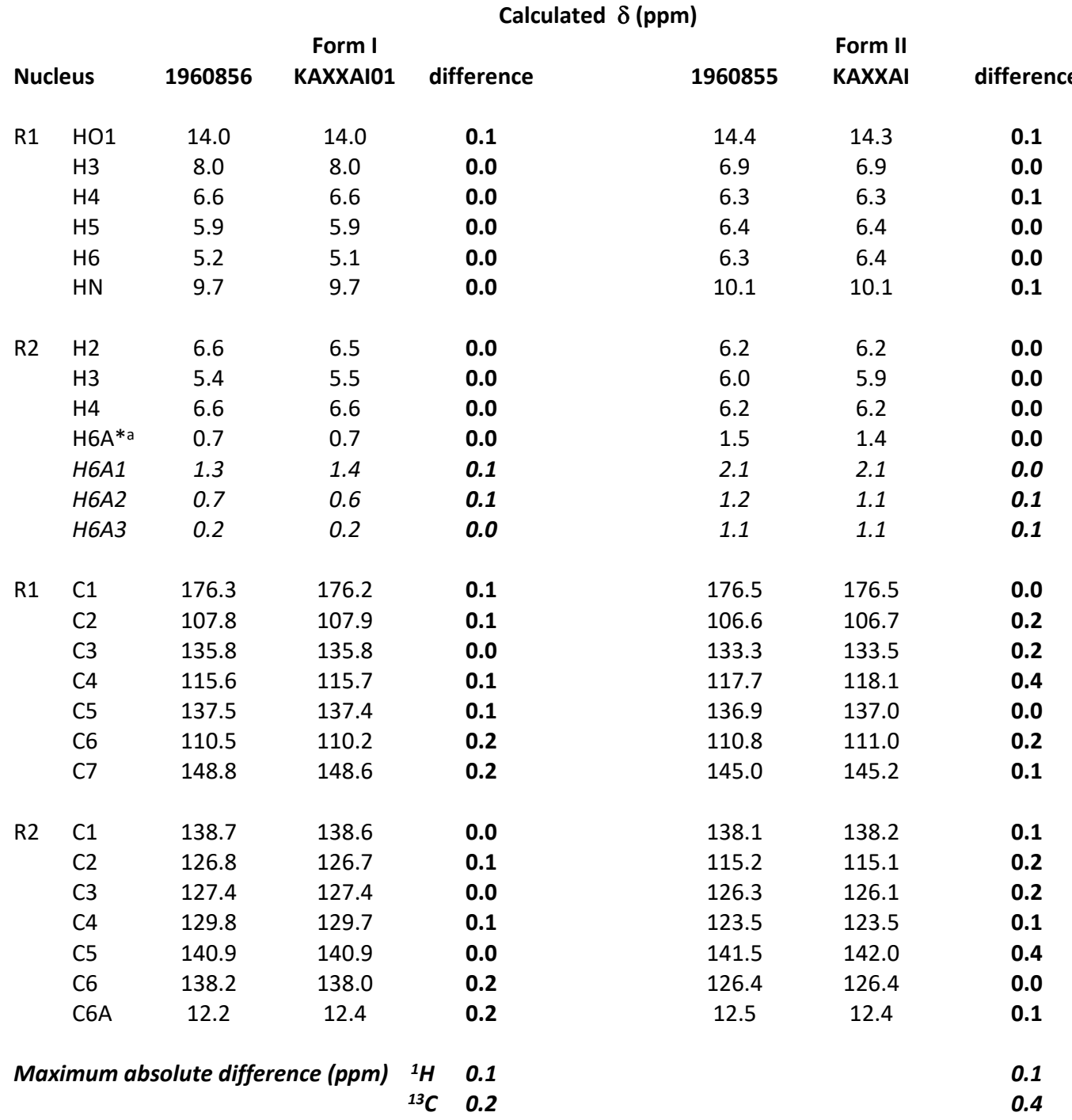

aMean chemical shift for methyl hydrogens, calculated from the individual chemical shifts (italics) from each crystal structure 
Supplementary Figure S2 - Overlay of structures before (coloured by element) and after (coloured by a block colour) geometry optimisation A) Form I (1960856). B) Form II (1960855). C) Form III (KAXXAI02). D) Form IV (KAXXAI03). RMSD $x$ was calculated using CSD Mercury using the software default settings with a $20 \%$ tolerance on distances and $20^{\circ}$ tolerance on angle over $\mathrm{x}$ number of molecules.

A

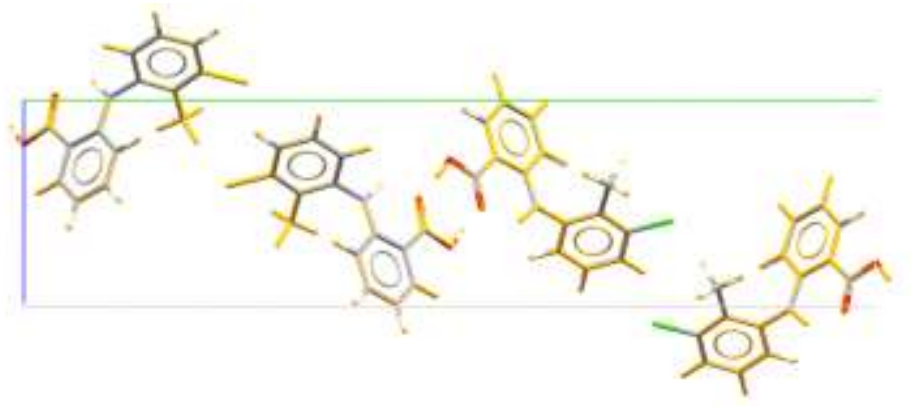

\section{Form I}

FAMSD

B

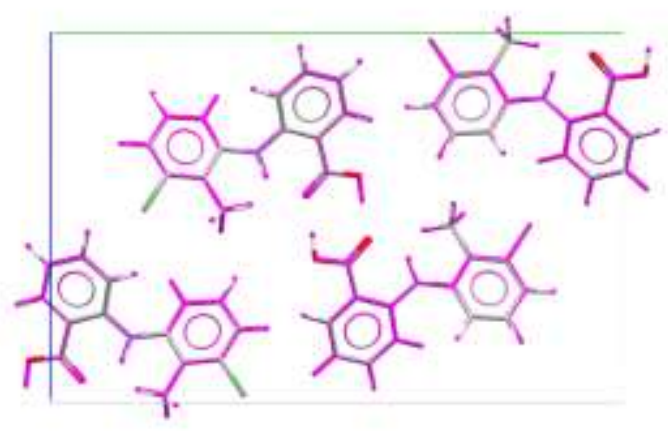

\section{Form II}

C

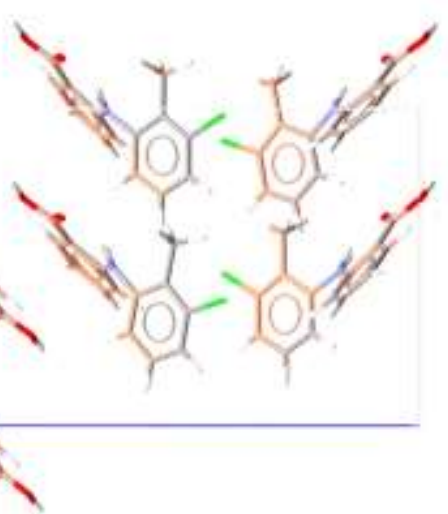

\section{Form III}

\section{Form IV}

D

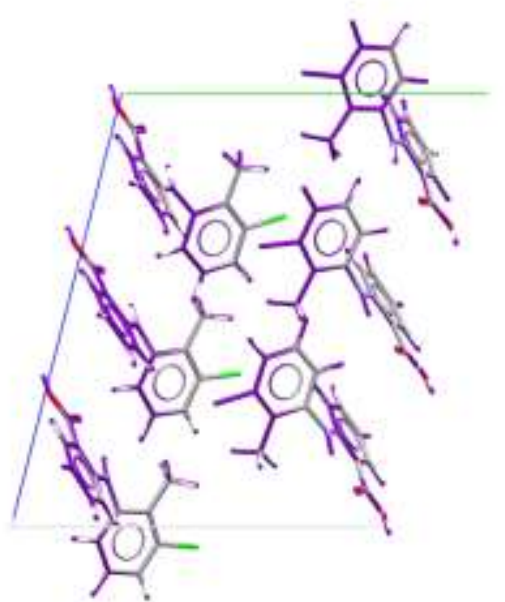


Supplementary Table S2 - Conformational behaviour at each rotatable bond in tolfenamic acid

$\begin{array}{lccc}\text { Torsion }^{\text {b }} & \begin{array}{c}\text { Mean angle } \\ \left({ }^{\circ}\right)^{\mathrm{c}}\end{array} & \begin{array}{c}\text { Population } \\ (\%)^{\mathrm{c}}\end{array} & \begin{array}{c}\text { Libration } \\ \left({ }^{\circ}\right)^{\mathrm{c}}\end{array} \\ \text { R1 O1A - R1 C1 - R1 C2 - R1 C7 } & 0 \pm 0^{\mathrm{d}} & 50 \pm 0^{\mathrm{d}} & 5 \pm 3^{\mathrm{e}} \\ & 180 \pm 0^{\mathrm{d}} & 50 \pm 0^{\mathrm{d}} & 5 \pm 3^{\mathrm{e}} \\ \mathrm{R} & 180 \pm 0^{\mathrm{d}} & 100.0 \pm 0.5 & 5 \pm 3^{\mathrm{e}} \\ \mathrm{R} & & & \\ \text { R1 C7 - R1 N8 - R2 C1 - R2 C6 } & 134.5 \pm 1.1 & 40.7 \pm 3.0 & 15 \pm 3 \\ & -134.5 \pm 1.1 & 40.7 \pm 3.0 & 15 \pm 3 \\ & 69.8 \pm 4.4 & 9.3 \pm 3.0 & 15 \pm 3 \\ & -69.8 \pm 44 & 9.3 \pm 3.0 & 15 \pm 3\end{array}$

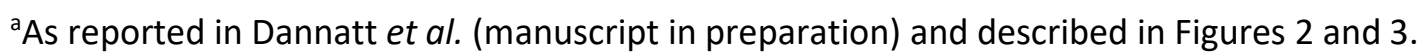

${ }^{\mathrm{b}}$ Refer to supplementary Figure $\mathrm{S} 3$ for atom and torsion definitions.

$c_{ \pm}$standard deviation

${ }^{d}$ Values and standard deviations dictated by symmetry considerations.

eEstimated values 
Supplementary Figure S3 - Definitions of the three torsions angles within TFA that can in principle affect the overall conformation are highlighted in yellow. Refer to Figure 1 for atom numbering.
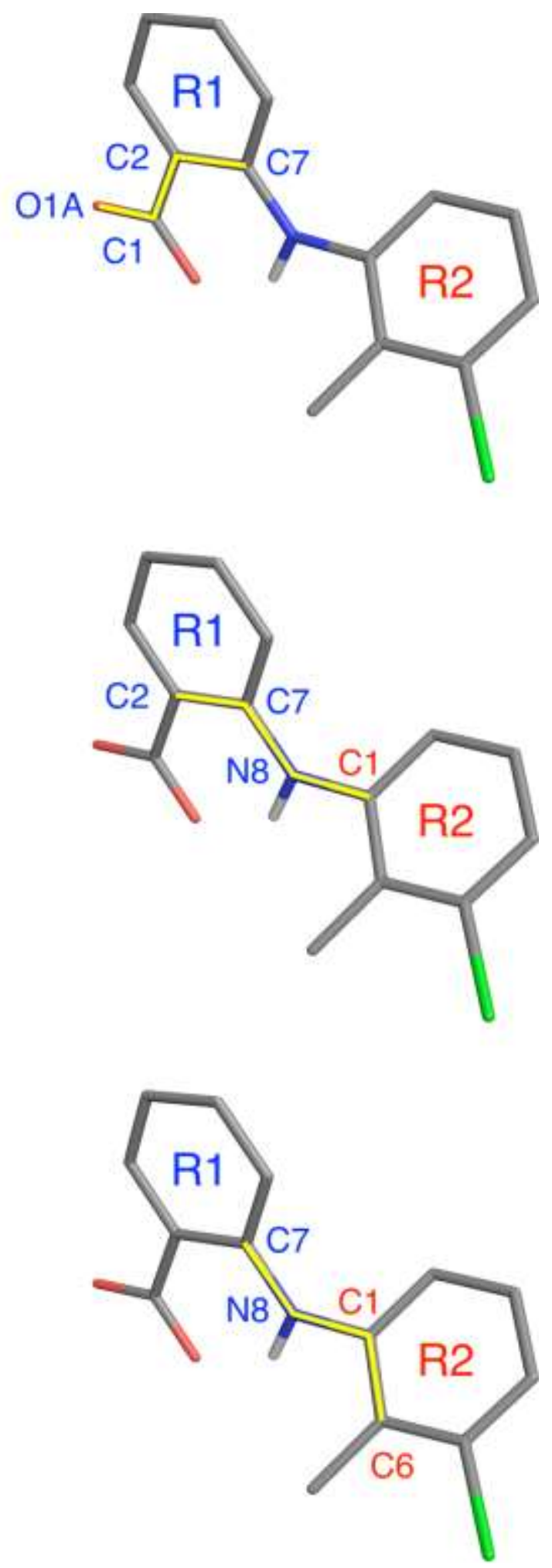
Supplementary Figure $\mathbf{S 4}$ - Comparison of crystal structures of TFA in order to assess 'base geometry' (i.e. bond lengths, bond angles and improper dihedrals). A) Overlay of Form I crystal structure conformations (KAXXAI01 and CSD accession code 1960856) showing that there are some differences in the positions of hydrogen atoms (arrows). B) Overlay of Form II crystal structure conformations (KAXXAl and CSD accession code 1960855) showing that there are some differences in the positions hydrogen atoms (one indicated by an arrow). C, D) Two views of an overlay of Form I and Form II crystal structure conformations (1960856 vs 1960855) showing the small differences in geometry principally around the nitrogen atom (R1 N8) by the angle subtended between the arrows.

A

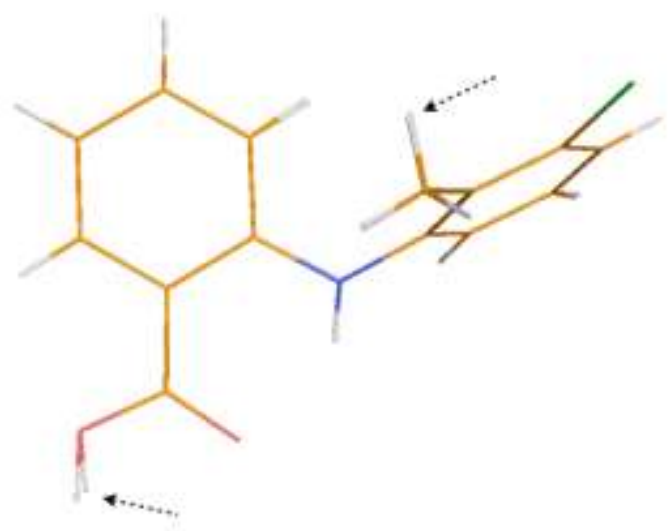

B

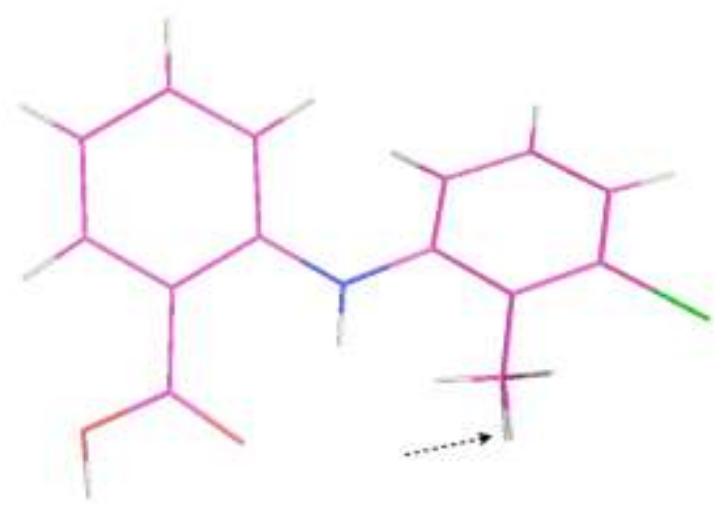

C

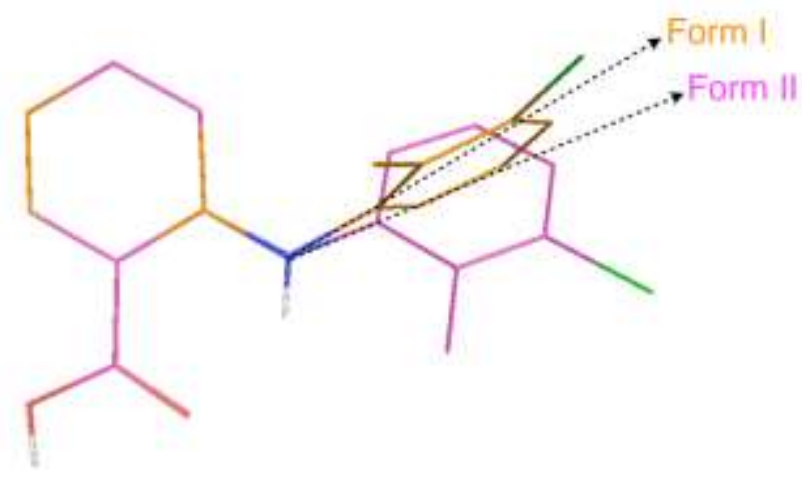

D

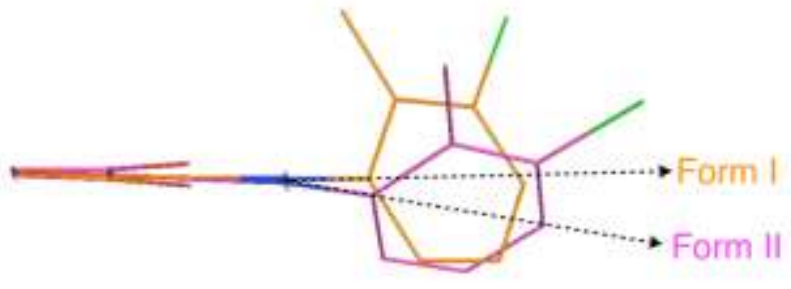




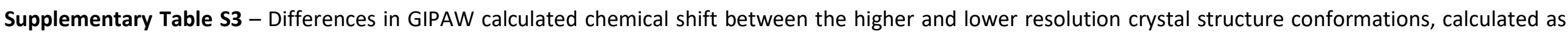
isolated single molecules (after geometry optimisation of the full crystal structures)

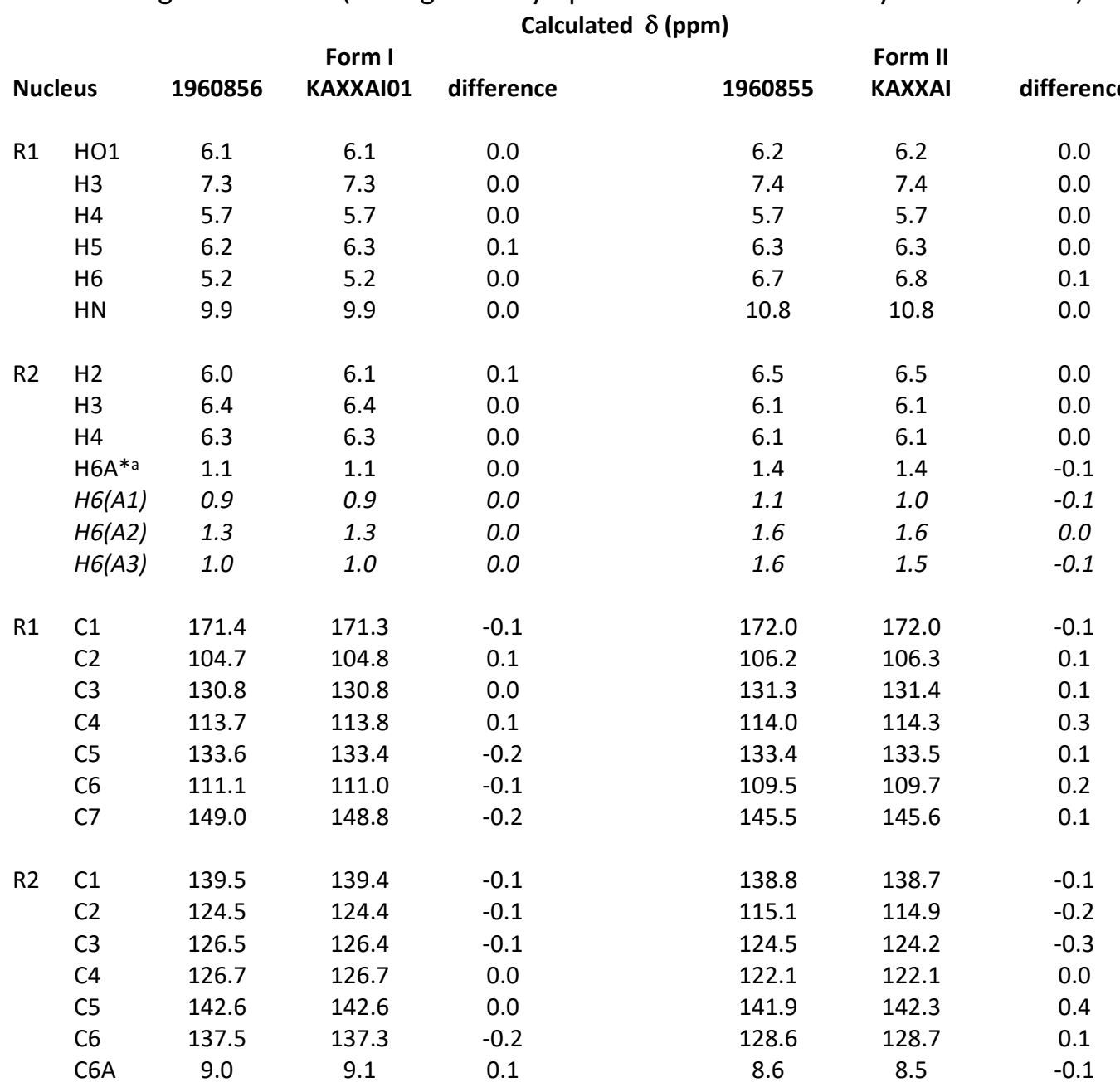

Maximum absolute difference (ppm) $\begin{array}{lll}{ }^{1} \mathrm{H} & 0.1 \\ { }^{13} \mathrm{C} & 0.2\end{array}$

${ }^{a}$ Mean chemical shift for methyl hydrogens, calculated from the individual chemical shifts (italics) from each crystal structure. 
Supplementary Table S4 - Calculated GIPAW chemical shifts for TFA as an isolated single molecule with R1 C7 - R1 N8 - R2 C1 - R2 C6 torsion angle set at $15^{\circ}$ intervals

Table S4A - Using geometry from symmetrised high-resolution Form I (CSD accession code 1960856)

\begin{tabular}{|c|c|c|c|c|c|c|c|c|c|c|c|c|c|c|c|c|c|c|c|c|c|c|c|c|c|c|}
\hline \multirow{2}{*}{\multicolumn{2}{|c|}{ Nucleus }} & \multicolumn{25}{|c|}{ Calculated chemical shift (ppm) at R1 C7 - R1 N8-R2 C1-R2 C6 torsion angle $\left({ }^{\circ}\right)$ of: } \\
\hline & & -180 & -165 & -150 & -135 & -120 & -105 & -90 & -75 & -60 & -45 & -30 & -15 & 0 & 15 & 30 & 45 & 60 & 75 & 90 & 105 & 120 & 135 & 150 & 165 & 180 \\
\hline \multirow[t]{6}{*}{ R1 } & HO1 & 4.80 & 4.80 & 4.77 & 4.77 & 4.73 & 4.73 & 4.70 & 4.73 & 4.77 & 4.77 & 4.80 & 4.80 & 4.73 & 4.80 & 4.80 & 4.77 & 4.77 & 4.70 & 4.70 & 4.73 & 4.73 & 4.77 & 4.77 & 4.83 & 4.80 \\
\hline & H3 & 7.67 & 7.60 & 7.50 & 7.47 & 7.40 & 7.37 & 7.37 & 7.43 & 7.43 & 7.47 & 7.50 & 7.50 & 7.53 & 7.50 & 7.50 & 7.47 & 7.43 & 7.43 & 7.37 & 7.40 & 7.40 & 7.47 & 7.53 & 7.60 & 7.67 \\
\hline & $\mathrm{H} 4$ & 5.90 & 5.93 & 5.87 & 5.80 & 5.73 & 5.70 & 5.70 & 5.77 & 5.80 & 5.83 & 5.83 & 5.83 & 5.80 & 5.83 & 5.83 & 5.83 & 5.77 & 5.73 & 5.70 & 5.70 & 5.73 & 5.80 & 5.87 & 5.90 & 5.90 \\
\hline & H5 & 6.63 & 6.60 & 6.47 & 6.43 & 6.33 & 6.27 & 6.27 & 6.33 & 6.43 & 6.50 & 6.50 & 6.57 & 6.57 & 6.57 & 6.53 & 6.47 & 6.43 & 6.37 & 6.27 & 6.27 & 6.37 & 6.43 & 6.47 & 6.60 & 6.63 \\
\hline & $\mathrm{H} 6$ & 7.20 & 7.30 & 7.13 & 6.77 & 6.13 & 5.43 & 5.03 & 5.23 & 5.43 & 5.63 & 5.90 & 6.20 & 1.77 & 6.23 & 5.87 & 5.60 & 5.40 & 5.23 & 5.03 & 5.43 & 6.13 & 6.77 & 7.13 & 7.30 & 7.20 \\
\hline & $\mathrm{HN}$ & 11.30 & 10.97 & 10.83 & 10.57 & 10.23 & 9.97 & 9.87 & 10.10 & 10.53 & 11.00 & 11.43 & 11.70 & 12.07 & 11.73 & 11.40 & 11.00 & 10.50 & 10.10 & 9.87 & 9.93 & 10.23 & 10.53 & 10.83 & 10.97 & 11.30 \\
\hline \multirow[t]{7}{*}{ R2 } & $\mathrm{H} 2$ & 6.90 & 6.90 & 6.70 & 6.53 & 6.30 & 6.20 & 6.17 & 6.13 & 6.03 & 6.00 & 6.00 & 5.97 & 6.03 & 5.97 & 5.97 & 6.03 & 6.07 & 6.07 & 6.10 & 6.17 & 6.33 & 6.50 & 6.70 & 6.87 & 6.90 \\
\hline & $\mathrm{H} 3$ & 6.27 & 6.23 & 6.20 & 6.23 & 6.30 & 6.40 & 6.43 & 6.40 & 6.37 & 6.33 & 6.27 & 6.23 & 6.20 & 6.27 & 6.27 & 6.30 & 6.37 & 6.43 & 6.43 & 6.43 & 6.30 & 6.23 & 6.20 & 6.23 & 6.27 \\
\hline & $\mathrm{H} 4$ & 6.00 & 6.07 & 6.07 & 6.17 & 6.27 & 6.40 & 6.50 & 6.43 & 6.30 & 6.23 & 6.10 & 6.03 & 6.03 & 6.03 & 6.13 & 6.20 & 6.30 & 6.40 & 6.47 & 6.40 & 6.30 & 6.13 & 6.07 & 6.07 & 6.00 \\
\hline & $H 6 A^{* a}$ & 1.64 & 1.60 & 1.53 & 1.46 & 1.37 & 1.31 & 1.24 & 1.19 & 1.11 & 1.10 & 1.19 & 1.39 & 3.42 & 1.40 & 1.20 & 1.09 & 1.11 & 1.18 & 1.23 & 1.28 & 1.34 & 1.46 & 1.53 & 1.59 & 1.64 \\
\hline & HGA1 & 1.20 & 2.43 & 2.23 & 2.00 & 1.53 & 1.20 & 1.03 & 0.93 & 1.00 & 1.13 & 1.30 & 1.47 & 1.80 & 1.53 & 1.47 & 1.43 & 1.40 & 1.23 & 1.07 & 1.00 & 1.03 & 1.17 & 1.23 & 1.27 & 1.20 \\
\hline & H6A2 & 1.20 & 1.27 & 1.23 & 1.17 & 1.07 & 1.07 & 1.07 & 1.27 & 1.40 & 1.43 & 1.43 & 1.53 & 1.60 & 1.47 & 1.30 & 1.13 & 1.00 & 0.97 & 1.00 & 1.23 & 1.53 & 2.03 & 2.27 & 2.43 & 1.20 \\
\hline & HGA3 & 2.53 & 1.10 & 1.13 & 1.20 & 1.50 & 1.67 & 1.63 & 1.37 & 0.93 & 0.73 & 0.83 & 1.17 & 6.87 & 1.20 & 0.83 & 0.70 & 0.93 & 1.33 & 1.63 & 1.60 & 1.47 & 1.17 & 1.10 & 1.07 & 2.53 \\
\hline \multirow[t]{7}{*}{ R1 } & $\mathrm{C} 1$ & 170.27 & 170.33 & 170.33 & 170.30 & 170.37 & 170.40 & 170.33 & 170.17 & 170.20 & 170.40 & 170.63 & 170.77 & 170.87 & 170.80 & 170.63 & 170.40 & 170.17 & 170.13 & 170.30 & 170.37 & 170.33 & 170.30 & 170.33 & 170.33 & 170.27 \\
\hline & C2 & & 107.67 & & 106.50 & 105.90 & 05.17 & 4.73 & 05.50 & & & & & & 107. & 106.73 & 106.23 & 105.8 & 105.40 & 104.73 & 105.17 & 105.87 & 06.50 & 7.03 & 107.63 & 107.77 \\
\hline & $\mathrm{C} 3$ & 133.47 & 132.87 & 132.63 & 132.33 & 131.97 & 131.67 & 131.63 & 131.90 & 131.90 & 131.80 & 131. & 131.93 & 132.57 & 131.93 & 131.77 & 131.80 & 131.90 & 131.87 & 131.63 & 131.67 & 131.97 & 132.37 & 132.63 & 132.90 & 133.47 \\
\hline & C4 & 115.23 & 115.70 & 115.60 & 115.27 & 114.60 & 113.73 & 113.37 & 114.10 & 114.73 & 115.1 & 115.3 & 115.3 & 115.27 & 115.33 & 115.27 & 115.07 & 114.70 & 114.07 & 113.37 & 113.73 & 114.57 & 115.27 & 115.60 & 115.70 & 115.23 \\
\hline & C5 & 135.20 & 134.67 & 134.37 & 134.27 & 134.27 & 134.23 & 134.30 & 134.33 & 134.07 & 133.67 & 133.30 & 133.27 & 133.80 & 133.23 & 133.27 & 133.63 & 134.07 & 134.37 & 134.30 & 134.27 & 134.27 & 134.30 & 134.37 & 134.67 & 135.20 \\
\hline & $\mathrm{C} 6$ & 113.03 & 111.77 & 110.00 & 109.07 & 109.33 & 110.17 & 110.63 & 110.87 & 111.57 & 112.67 & 114.53 & 116.70 & 117.33 & 116.67 & 114.53 & 112.70 & 111.47 & 110.80 & 110.67 & 110.17 & 109.37 & 109.10 & 109.97 & 111.77 & 113.03 \\
\hline & C7 & 146.27 & 145.77 & 145.93 & 147.13 & 149.17 & 150.93 & 151.37 & 150.03 & 147.93 & 146.33 & 145.20 & 144.50 & 144.53 & 144.50 & 145.20 & 146.30 & 147.93 & 150.00 & 151.33 & 150.90 & 149.17 & 147.10 & 145.97 & 145.73 & 146.27 \\
\hline \multirow[t]{7}{*}{ R2 } & C1 & 141.23 & 140.87 & 1 & 1 & 139. & 139. & & 139. & & & & & & & & & & 139 & 93 & & 57 & & & & 141.23 \\
\hline & $\mathrm{C} 2$ & 110.60 & 110.90 & 113.83 & 118.37 & 123.67 & 127.60 & 128.27 & 124.87 & 121.77 & 119.90 & 118.97 & 118.97 & 119.83 & 119.00 & 118.93 & 119.77 & 121.77 & 124.90 & 128.20 & 127.57 & 123.67 & 118.33 & 113.80 & 110.83 & 110.60 \\
\hline & C3 & 125.17 & 124.73 & 124.40 & & 124.87 & & & & & & & & & & & & & 126.20 & & 125.70 & & 124.40 & & 124.73 & 125.17 \\
\hline & C4 & 120.63 & 121.20 & 122.3 & 123. & 125. & 127. & 128. & 1 & & & & & & & 122 & 123 & 125 & 0 & 47 & 57 & 73 & 87 & 30 & 20 & 120.63 \\
\hline & C5 & & 141.7 & & & & & & & & & & & & & & & & & 143 & 143 & 33 & 142.43 & 00 & 141.70 & 143.50 \\
\hline & $\mathrm{C} 6$ & 126.23 & 125.87 & 127.17 & 129.93 & 133.97 & 138.10 & 140.43 & 138.20 & 133.70 & 128.87 & 124.17 & 120.27 & 117.97 & 120.27 & 124.20 & 128.83 & 133.73 & 138.20 & 140.43 & 138.10 & 134.03 & 129.93 & 127.13 & 125.87 & 126.23 \\
\hline & C6A & 8.47 & 9.07 & 8.70 & 8.70 & 8.87 & 9.00 & 8.90 & 9.20 & 11.27 & 13.73 & 15.83 & 17.53 & 18.67 & 17.57 & 15.83 & 13.73 & 11.23 & 9.17 & 8.87 & 8.97 & 8.87 & 8.70 & 8.67 & 9.03 & 8.47 \\
\hline
\end{tabular}

${ }^{a}$ Mean chemical shift for methyl hydrogens, calculated from the individual chemical shifts (italics) from each crystal structure. 
Supplementary Table S4 - Calculated chemical shifts for TFA in an isolated box with R1 C7 - R1 N8 - R2 C1 - R2 C6 torsion angle set at $15^{\circ}$ intervals

Table S4B - Using geometry of symmetrised high-resolution Form 2 (CSD accession code 1960855)

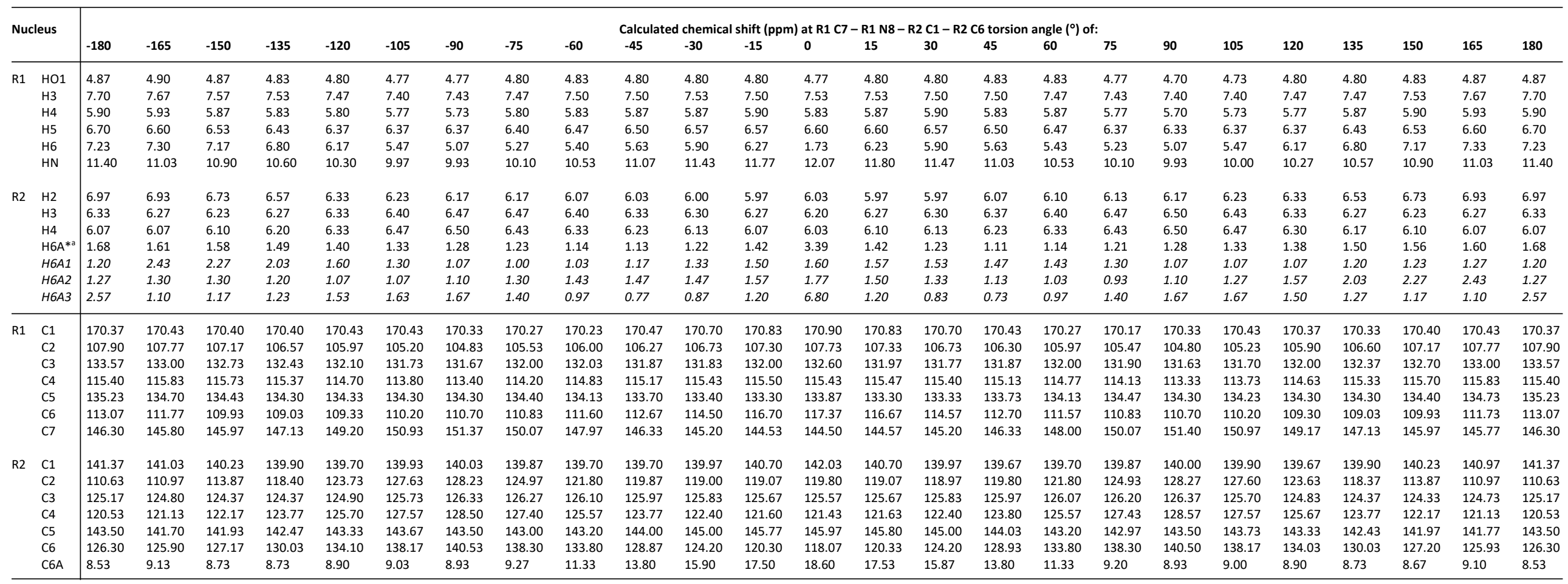

aMean chemical shift for methyl hydrogens, calculated from the individual chemical shifts (italics) from each crystal structure. 
Supplementary Table S4 - Calculated chemical shifts for TFA in an isolated box with R1 C7 - R1 N8 - R2 C1 - R2 C6 torsion angle set at $15^{\circ}$ intervals

Table S4C - Difference in calculated chemical shift between using geometry of symmetrised high-resolution Form 1 and Form 2 (CSD accession codes 1960856 and 1960855$)$

\begin{tabular}{|c|c|c|c|c|c|c|c|c|c|c|c|c|c|c|c|c|c|c|c|c|c|c|c|c|c|c|}
\hline \multirow{2}{*}{\multicolumn{2}{|c|}{ Nucleus }} & \multicolumn{25}{|c|}{ Difference in calculated chemical shift (Form $2-$ Form $1 ;$ ppm) at R1 C7 - R1 N8 - R2 C1-R2 C6 torsion angle ( (') of: } \\
\hline & & -180 & -165 & -150 & -135 & -120 & -105 & -90 & -75 & -60 & -45 & -30 & -15 & 0 & 15 & 30 & 45 & 60 & 75 & 90 & 105 & 120 & 135 & 150 & 165 & 180 \\
\hline \multirow{6}{*}{ R1 } & HO1 & 0.07 & 0.10 & 0.10 & 0.07 & 0.07 & 0.03 & 0.07 & 0.07 & 0.07 & 0.03 & 0.00 & 0.00 & 0.03 & 0.00 & 0.00 & 0.07 & 0.07 & 0.07 & 0.00 & 0.00 & 0.07 & 0.03 & 0.07 & 0.03 & 0.07 \\
\hline & $\mathrm{H3}$ & 0.03 & 0.07 & 0.07 & 0.07 & 0.07 & 0.03 & 0.07 & 0.03 & 0.07 & 0.03 & 0.03 & 0.00 & 0.00 & 0.03 & 0.00 & 0.03 & 0.03 & 0.00 & 0.03 & 0.00 & 0.07 & 0.00 & 0.00 & 0.07 & 0.03 \\
\hline & $\mathrm{H} 4$ & 0.00 & 0.00 & 0.00 & 0.03 & 0.07 & 0.07 & 0.03 & 0.03 & 0.03 & 0.03 & 0.03 & 0.07 & 0.03 & 0.03 & 0.07 & 0.00 & 0.10 & 0.03 & 0.00 & 0.03 & 0.03 & 0.07 & 0.03 & 0.03 & 0.00 \\
\hline & H5 & 0.07 & 0.00 & 0.07 & 0.00 & 0.03 & 0.10 & 0.10 & 0.07 & 0.03 & 0.00 & 0.07 & 0.00 & 0.03 & 0.03 & 0.03 & 0.03 & 0.03 & 0.00 & 0.07 & 0.10 & 0.00 & 0.00 & 0.07 & 0.00 & 0.07 \\
\hline & $\mathrm{H} 6$ & 0.03 & 0.00 & 0.03 & 0.03 & 0.03 & 0.03 & 0.03 & 0.03 & 0.03 & 0.00 & 0.00 & 0.07 & 0.03 & 0.00 & 0.03 & 0.03 & 0.03 & 0.00 & 0.03 & 0.03 & 0.03 & 0.03 & 0.03 & 0.03 & 0.03 \\
\hline & $\mathrm{HN}$ & 0.10 & 0.07 & 0.07 & 0.03 & 0.07 & 0.00 & 0.07 & 0.00 & 0.00 & 0.07 & 0.00 & 0.07 & 0.00 & 0.07 & 0.07 & 0.03 & 0.03 & 0.00 & 0.07 & 0.07 & 0.03 & 0.03 & 0.07 & 0.07 & 0.10 \\
\hline \multirow[t]{4}{*}{ R2 } & $\mathrm{H} 2$ & 0.07 & 0.03 & 0.03 & 0.03 & 0.03 & 0.03 & 0.00 & 0.03 & 0.03 & 0.03 & 0.00 & 0.00 & 0.00 & 0.00 & 0.00 & 0.03 & 0.03 & 0.07 & 0.07 & 0.07 & 0.00 & 0.03 & 0.03 & 0.07 & 0.07 \\
\hline & H3 & 0.07 & 0.03 & 0.03 & 0.03 & 0.03 & 0.00 & 0.03 & 0.07 & 0.03 & 0.00 & 0.03 & 0.03 & 0.00 & 0.00 & 0.03 & 0.07 & 0.03 & 0.03 & 0.07 & 0.00 & 0.03 & 0.03 & 0.03 & 0.03 & 0.07 \\
\hline & $\mathrm{H} 4$ & 0.07 & 0.00 & 0.03 & 0.03 & 0.07 & 0.07 & 0.00 & 0.00 & 0.03 & 0.00 & 0.03 & 0.03 & 0.00 & 0.07 & 0.00 & 0.03 & 0.03 & 0.03 & 0.03 & 0.07 & 0.00 & 0.03 & 0.03 & 0.00 & 0.07 \\
\hline & $\mathrm{H} 6 \mathrm{~A}^{*}$ & 0.03 & 0.01 & 0.04 & 0.03 & 0.03 & 0.02 & 0.03 & 0.04 & 0.03 & 0.03 & 0.03 & 0.03 & 0.03 & 0.02 & 0.03 & 0.02 & 0.03 & 0.03 & 0.04 & 0.06 & 0.03 & 0.04 & 0.02 & 0.01 & 0.03 \\
\hline \multirow[t]{7}{*}{ R1 } & C1 & 0.10 & 0.10 & 0.07 & 0.10 & 0.07 & 0.03 & 0.00 & 0.10 & 0.03 & 0.07 & 0.07 & 0.07 & 0.03 & 0.03 & 0.07 & 0.03 & 0.10 & 0.03 & 0.03 & 0.07 & 0.03 & 0.03 & 0.07 & 0.10 & 0.10 \\
\hline & C2 & 0.13 & 0.10 & 0.17 & 0.07 & 0.07 & 0.03 & 0.10 & 0.03 & 0.07 & 0.03 & 0.03 & 0.00 & 0.00 & 0.03 & 0.00 & 0.07 & 0.10 & 0.07 & 0.07 & 0.07 & 0.03 & 0.10 & 0.13 & 0.13 & 0.13 \\
\hline & C3 & 0.10 & 0.13 & 0.10 & 0.10 & 0.13 & 0.07 & 0.03 & 0.10 & 0.13 & 0.07 & 0.07 & 0.07 & 0.03 & 0.03 & 0.00 & 0.07 & 0.10 & 0.03 & 0.00 & 0.03 & 0.03 & 0.00 & 0.07 & 0.10 & 0.10 \\
\hline & C4 & 0.17 & 0.13 & 0.13 & 0.10 & 0.10 & 0.07 & 0.03 & 0.10 & 0.10 & 0.07 & 0.13 & 0.17 & 0.17 & 0.13 & 0.13 & 0.07 & 0.07 & 0.07 & 0.03 & 0.00 & 0.07 & 0.07 & 0.10 & 0.13 & 0.17 \\
\hline & C5 & 0.03 & 0.03 & 0.07 & 0.03 & 0.07 & 0.07 & 0.00 & 0.07 & 0.07 & 0.03 & 0.10 & 0.03 & 0.07 & 0.07 & 0.07 & 0.10 & 0.07 & 0.10 & 0.00 & 0.03 & 0.03 & 0.00 & 0.03 & 0.07 & 0.03 \\
\hline & C6 & 0.03 & 0.00 & 0.07 & 0.03 & 0.00 & 0.03 & 0.07 & 0.03 & 0.03 & 0.00 & 0.03 & 0.00 & 0.03 & 0.00 & 0.03 & 0.00 & 0.10 & 0.03 & 0.03 & 0.03 & 0.07 & 0.07 & 0.03 & 0.03 & 0.03 \\
\hline & C7 & 0.03 & 0.03 & 0.03 & 0.00 & 0.03 & 0.00 & 0.00 & 0.03 & 0.03 & 0.00 & 0.00 & 0.03 & 0.03 & 0.07 & 0.00 & 0.03 & 0.07 & 0.07 & 0.07 & 0.07 & 0.00 & 0.03 & 0.00 & 0.03 & 0.03 \\
\hline \multirow[t]{7}{*}{ R2 } & $\mathrm{C} 1$ & 0.13 & 0.17 & 0.07 & 0.07 & 0.10 & 0.10 & 0.10 & 0.07 & 0.10 & 0.07 & 0.07 & 0.10 & 0.03 & 0.03 & 0.10 & 0.07 & 0.10 & 0.17 & 0.07 & 0.07 & 0.10 & 0.03 & 0.07 & 0.10 & 0.13 \\
\hline & C2 & 0.03 & 0.07 & 0.03 & 0.03 & 0.07 & 0.03 & 0.03 & 0.1 & 0.03 & 0.03 & 0.03 & 0.10 & 0.03 & 0.07 & 0.03 & 0.03 & 0.03 & 0.03 & 0.07 & 0.03 & 0.03 & 0.03 & 0.07 & 0.13 & 0.03 \\
\hline & C3 & 0.00 & 0.07 & 0.03 & 0.03 & 0.03 & 0.03 & 0.00 & 0.07 & 0.07 & 0.00 & 0.00 & 0.03 & 0.07 & 0.00 & 0.00 & 0.03 & 0.03 & 0.00 & 0.03 & 0.00 & 0.07 & 0.03 & 0.03 & 0.00 & 0.00 \\
\hline & $\mathrm{C} 4$ & 0.10 & 0.07 & 0.13 & 0.07 & 0.00 & 0.03 & 0.00 & 0.03 & 0.03 & 0.03 & 0.07 & 0.07 & 0.13 & 0.03 & 0.03 & 0.00 & 0.03 & 0.03 & 0.10 & 0.00 & 0.07 & 0.10 & 0.13 & 0.07 & 0.10 \\
\hline & C5 & 0.00 & 0.03 & 0.07 & 0.03 & 0.03 & 0.03 & 0.07 & 0.03 & 0.03 & 0.03 & 0.03 & 0.00 & 0.00 & 0.03 & 0.03 & 0.03 & 0.0 & 0.0 & 0.8 & 0.10 & 0.00 & 0.00 & 0.03 & 0.07 & 0.00 \\
\hline & C6 & 0.07 & 0.03 & 0.00 & 0.10 & 0.13 & 0.07 & 0.10 & 0.1 & 0.10 & 0.00 & 0.03 & 0.03 & 0.10 & 0.07 & 0.00 & 0.10 & 0.07 & 0.10 & 0.07 & 0.07 & 0.00 & 0.10 & 0.07 & 0.07 & 0.07 \\
\hline & C6A & 0.07 & 0.07 & 0.03 & 0.03 & 0.03 & 0.03 & 0.03 & 0.07 & 0.07 & 0.07 & 0.07 & 0.03 & 0.07 & 0.03 & 0.03 & 0.07 & 0.10 & 0.03 & 0.07 & 0.03 & 0.03 & 0.03 & 0.00 & 0.07 & 0.07 \\
\hline
\end{tabular}

Absolute difference (ppm)

Maximum (bold red)

Mean

Standard deviation

$\begin{array}{cc}{ }^{1} \mathbf{H}^{\mathrm{a}} & { }^{13} \mathbf{C} \\ 0.10 & 0.17 \\ 0.04 & 0.06 \\ 0.03 & 0.04\end{array}$

${ }^{\text {aValues for }}{ }^{1} \mathrm{H}$ calculated for all hydrogens excluding R1 HN because it is not used in the scoring function (see text). 
Supplementary Table S4 - Calculated chemical shifts for TFA in an isolated box with R1 C7 - R1 N8 - R2 C1 - R2 C6 torsion angle set at $15^{\circ}$ intervals

Table S4D - Mean calculated chemical shifts using geometry of symmetrised high-resolution Form 1 and Form 2 (CSD accession codes 1960856 and 1960855 )

\begin{tabular}{|c|c|c|c|c|c|c|c|c|c|c|c|c|c|c|c|c|c|c|c|c|c|c|c|c|c|c|}
\hline \multirow{2}{*}{\multicolumn{2}{|c|}{ Nucleus }} & \multirow{3}{*}{$\begin{array}{l}-180 \\
4.83\end{array}$} & \multirow{3}{*}{$\begin{array}{l}-165 \\
4.85\end{array}$} & \multirow{3}{*}{$\begin{array}{l}-150 \\
4.82\end{array}$} & \multirow{3}{*}{$\begin{array}{l}-135 \\
4.80\end{array}$} & \multirow{3}{*}{$\begin{array}{l}-120 \\
4.77\end{array}$} & \multirow{3}{*}{$\begin{array}{l}-105 \\
4.75\end{array}$} & \multirow{3}{*}{$\begin{array}{l}-90 \\
4.73\end{array}$} & \multirow{3}{*}{$\begin{array}{l}-75 \\
4.77\end{array}$} & \multirow{3}{*}{$\begin{array}{l}-60 \\
4.80\end{array}$} & \multicolumn{8}{|c|}{ emical shift at R1 C7 - R1 N8-R2 C1-R2 C6 tor } & \multirow[b]{2}{*}{75} & \multirow[b]{2}{*}{90} & \multirow[b]{2}{*}{105} & \multirow[b]{2}{*}{120} & \multirow[b]{2}{*}{135} & \multirow[b]{2}{*}{150} & \multirow[b]{2}{*}{165} & \multirow[b]{2}{*}{180} \\
\hline & & & & & & & & & & & -45 & -30 & -15 & 0 & 15 & 30 & 45 & 60 & & & & & & & & \\
\hline \multirow[t]{6}{*}{ R1 } & HO1 & & & & & & & & & & 4.78 & 4.80 & 4.80 & 4.75 & 4.80 & 4.80 & 4.80 & 4.80 & 4.73 & 4.70 & 4.73 & 4.77 & 4.78 & 4.80 & 4.85 & 4.83 \\
\hline & H3 & 7.68 & 7.63 & 7.53 & 7.50 & 7.43 & 7.38 & 7.40 & 7.45 & 7.47 & 7.48 & 7.52 & 7.50 & 7.53 & 7.52 & 7.50 & 7.48 & 7.45 & 7.43 & 7.38 & 7.40 & 7.43 & 7.47 & 7.53 & 7.63 & 7.68 \\
\hline & $\mathrm{H} 4$ & 5.90 & 5.93 & 5.87 & 5.82 & 5.77 & 5.73 & 5.7 & & & 5.85 & 5.85 & 5.87 & 5.82 & 5.85 & 5.87 & 5.83 & 5.8 & & 5.7 & 5.7 & & & 5.88 & 5.92 & 5.90 \\
\hline & H5 & 6.67 & & & & & & & & & & 6.5 & 6.57 & & 58 & 55 & 6. & & 6. & 6.30 & 6.32 & 37 & & 6.50 & 6.60 & 6.67 \\
\hline & $\mathrm{H} 6$ & 7.22 & 7.30 & 7.15 & 6.78 & 6.15 & 5.45 & 5.05 & 5.25 & 5.42 & 5.63 & 5.90 & 6.23 & 1.75 & 6.23 & 5.88 & 5.62 & 5.42 & 5.23 & 5.05 & 5.45 & 6.15 & 6.78 & 7.15 & 7.32 & 7.22 \\
\hline & $\mathrm{HN}$ & 11.35 & 11.00 & 10.87 & 10.58 & 10.27 & 9.97 & 9.90 & 10.10 & 10.53 & 11.03 & 11.43 & 11.73 & 12.07 & 11.77 & 11.43 & 11.02 & 10.52 & 10.10 & 9.90 & 9.97 & 10.25 & 10.55 & 10.87 & 11.00 & 11.35 \\
\hline \multirow[t]{4}{*}{ R2 } & $\mathrm{H} 2$ & 6.93 & 6.92 & 6.72 & 6.55 & 6.32 & 6.22 & & & 605 & & 6.00 & 5.97 & & 5.97 & 5.9 & 6.0 & 6.0 & 6.11 & 6.13 & 6.20 & 6.33 & 6.52 & 6.72 & 6.90 & 6.93 \\
\hline & H3 & 6.30 & 6.25 & 6.22 & 6.25 & 6.32 & 6.40 & 6.45 & 6.43 & 6.38 & 6.33 & 6.28 & 6.25 & 6.20 & 6.27 & 6.28 & 6.33 & 6.38 & 6.45 & 6.47 & 6.43 & 6.32 & 6.25 & 6.22 & 6.25 & 6.30 \\
\hline & $\mathrm{H} 4$ & 6.03 & 6.07 & 6.08 & 6.18 & 6.30 & 6.43 & 6.50 & 6.43 & 6.32 & 6.23 & 6.12 & 6.05 & 6.03 & 6.07 & 6.13 & 6.22 & 6.32 & 6.42 & 6.48 & 6.43 & 6.30 & 6.15 & 6.08 & 6.07 & 6.03 \\
\hline & $\mathrm{H}^{\prime} \mathrm{A}^{*}$ & 1.66 & 1.61 & 1.56 & 1.47 & 1.38 & 1.32 & 1.26 & 1.21 & 1.13 & 1.12 & 1.21 & 1.41 & 3.41 & 1.41 & 1.22 & 1.10 & 1.13 & 1.19 & 1.26 & 1.31 & 1.36 & 1.48 & 1.54 & 1.59 & 1.66 \\
\hline \multirow[t]{7}{*}{ R1 } & C1 & 170.32 & 170.38 & 170.37 & 170.35 & 170.40 & 170.42 & 170.33 & 170.22 & 170.22 & & 170.67 & 170.80 & 170.88 & 170.82 & 170.67 & 170.42 & 170.22 & 170.15 & 170.32 & 170.40 & 170.35 & 170.32 & 170.37 & 170.38 & 170.32 \\
\hline & $\mathrm{C} 2$ & 107.83 & 107.72 & 107.08 & 6.53 & 05.93 & 105.18 & 104.78 & 105.52 & 105.97 & 106.25 & 106.72 & 107.30 & 107.73 & 107.32 & 106.73 & 106.27 & 105.92 & 105.43 & 104.77 & 105.20 & 105.88 & 106.55 & 107.10 & 107.70 & 107.83 \\
\hline & C3 & 133.52 & 132.93 & 132.68 & 2.38 & 32.03 & 31.70 & 131.65 & 131.95 & 131.97 & & & 131.97 & 132.58 & 131.95 & 131.77 & 131.83 & 131.95 & 131.88 & 131.63 & 131.68 & 131.98 & 132.37 & 132.67 & 132.95 & 133.52 \\
\hline & C4 & 115.32 & 115.77 & 1 & 5.32 & 4.65 & 3.77 & 3.38 & 14.15 & & & & & & 115.40 & & & 1 & 114.10 & 113.35 & 113.73 & 114.60 & & & 77 & 115.32 \\
\hline & C5 & 135.22 & 34.68 & & & 1 & & 1 & & & & & & & & & & 4. & 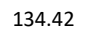 & 0 & 25 & 28 & & & 70 & 35.22 \\
\hline & C6 & 113.05 & 11.77 & 1 & 1 & 1 & 11 & 110.67 & 110.8 & & & & & 117.35 & 116.67 & 114.55 & 70 & 111. & 110.82 & 110.68 & 110.18 & 109.33 & 109.07 & 109.95 & 111.75 & 113.05 \\
\hline & C7 & 146.28 & 145.78 & 145.95 & 147.13 & 149.18 & 150.93 & 151.37 & 150.05 & 147.95 & 146.33 & 145.20 & 144.52 & 144.52 & 144.53 & 145.20 & 146.32 & 147.97 & 150.03 & 151.37 & 150.93 & 149.17 & 147.12 & 145.97 & 145.75 & 146.28 \\
\hline \multirow[t]{7}{*}{ R2 } & C1 & & & & & & & & & & & & & & & & & & & & & & & & & \\
\hline & $\mathrm{C} 2$ & & & & & & & & & & & & & & & & & & & & & & & & & 110.62 \\
\hline & C3 & & & & & & & & & & & & & & & & & & & & & & & & & 125 \\
\hline & C4 & 120.58 & 121.17 & 122.23 & 123.80 & 125.70 & 127.55 & 128.50 & 127.38 & 125.55 & & 122.43 & 121. & 121.50 & 121.65 & 122.42 & 123.80 & 125.55 & 127.42 & 128.52 & 127.57 & 125.70 & 123.82 & 122.23 & 121.17 & 120.58 \\
\hline & C5 & & 141.72 & 141.9 & 142.4 & 143.35 & 143.65 & 143.4 & 142.98 & & & & & & & & & & 142.97 & 143.48 & 143.68 & 143.33 & 142.43 & 141.98 & 141.73 & 143.50 \\
\hline & C6 & 126.27 & 125.88 & 127. & 129.9 & 134.03 & 138.1 & 140.4 & 138.25 & & & & & & & & & & & & & 134.03 & & 127.17 & 25.90 & 126.27 \\
\hline & C6A & 8.50 & 9.10 & 8.72 & 8.72 & 8.88 & 9.02 & 8.92 & 9.23 & 11.30 & 13.77 & 15.87 & 17.52 & 18.63 & 17.55 & & 13.77 & 11.28 & 9.18 & 8.90 & 8.98 & 8.88 & 8.72 & 8.67 & 9.07 & 8.50 \\
\hline
\end{tabular}


Supplementary Figure S5 - Variation of GIPAW calculated chemical shifts (left ${ }^{1} \mathrm{H}$, right ${ }^{13} \mathrm{C}$ ) with R1 C7 - R1 N8 - R2 C1 - R2 C6 torsion angle for TFA. Chemical shifts were calculated for every $15^{\circ}$ for the symmetrised high-resolution conformations of both Form I and II as gas-phase isolated molecules and the mean value taken to produce the graph points (see Table S4D). Nuclei are identified by blue and red labelling for $\mathrm{R} 1$ and $\mathrm{R} 2$, respectively.
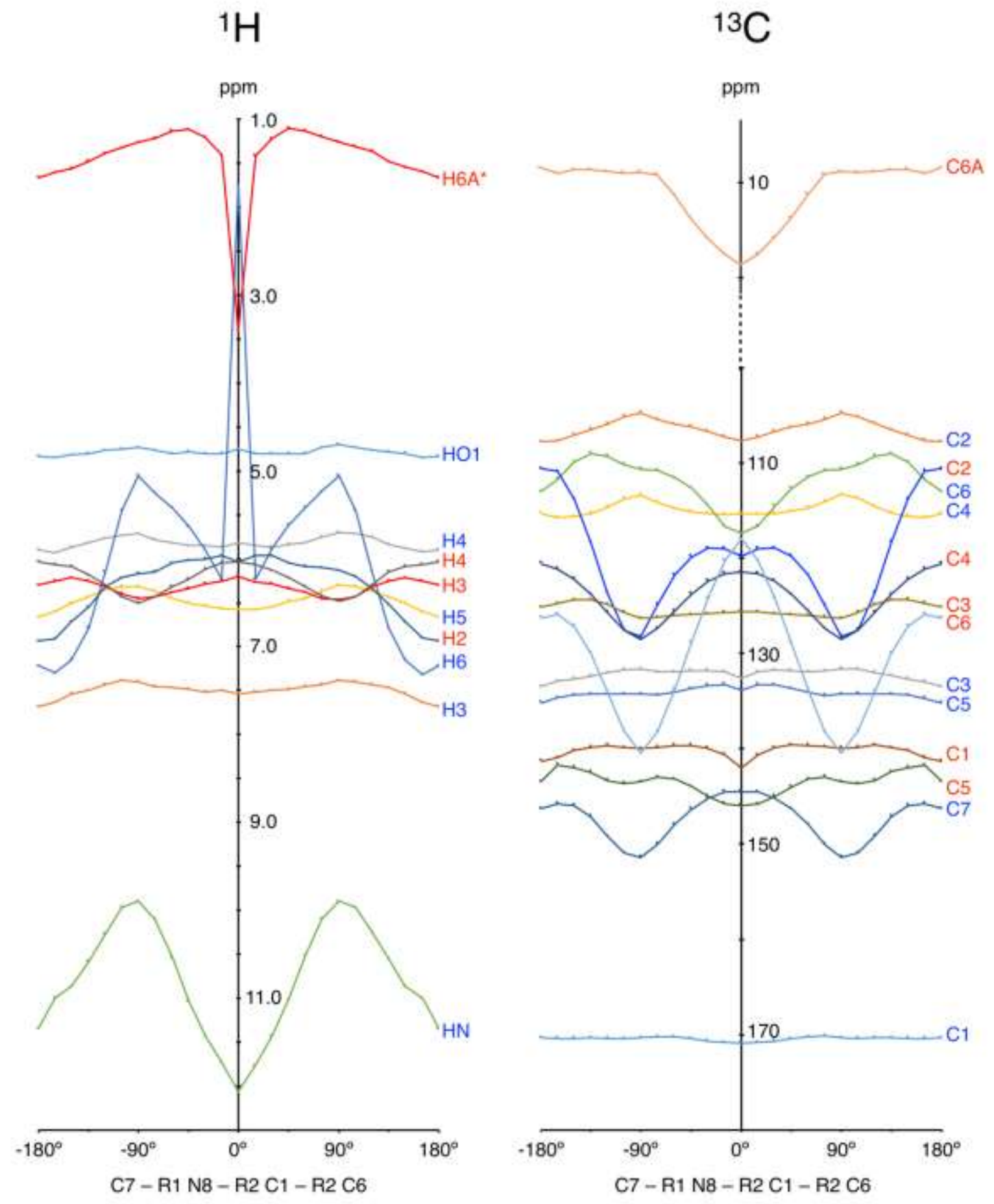
Supplementary Table S5 - Maximum absolute change in calculated GIPAW chemical shift for TFA as the R1 C7 - R1 N8 - R2 C1 - R2 C6 torsion varies between $-180^{\circ}$ to $180^{\circ}$ (see Figure S4)

$\begin{array}{ccc}\text { Nucleus } & \begin{array}{c}\text { Maximum absolute change } \\ \text { (ppm) }\end{array} \\ \text { R1 } & \text { H1 } & \\ \text { H3 } & 0.15 \\ \text { H4 } & 0.30 \\ \text { H5 } & 0.23 \\ \text { H6 } & 0.37 \\ \text { HN } & 5.57 \\ & & 2.17 \\ \text { R2 } & \text { H2 } & \\ \text { H3 } & 0.97 \\ \text { H4 } & 0.27 \\ \text { H6A } & 0.47 \\ & 2.31 \\ \text { R1 } 1 & \\ \text { C2 } & 0.73 \\ \text { C3 } & 3.07 \\ \text { C4 } & 1.88 \\ \text { C5 } & 2.42 \\ \text { C6 } & 1.95 \\ \text { C7 } & 8.30 \\ \text { R2 } & 6.85 \\ \text { C1 } & \\ \text { C2 } & 2.43 \\ \text { C3 } & 17.63 \\ \text { C4 } & 2.00 \\ \text { C5 } & 7.93 \\ \text { C6 } & 4.25 \\ \text { C6A } & 22.47 \\ & 10.13 \\ & \end{array}$

a Values calculated from Table S4D. 
Supplementary Table $\mathbf{S 6}$ - Calculation of $\delta_{\text {solution calc }}$ values for TFA by combining measured solution population occupancies and calculated chemical shifts for isolated box conformations at $15^{\circ}$ intervals

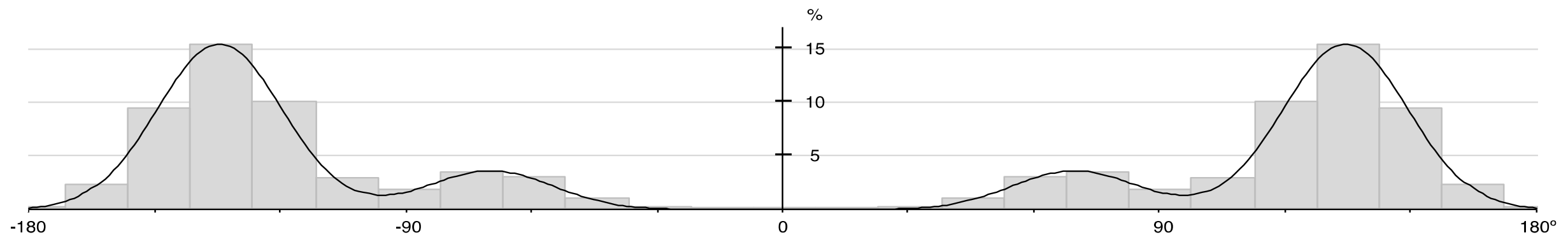

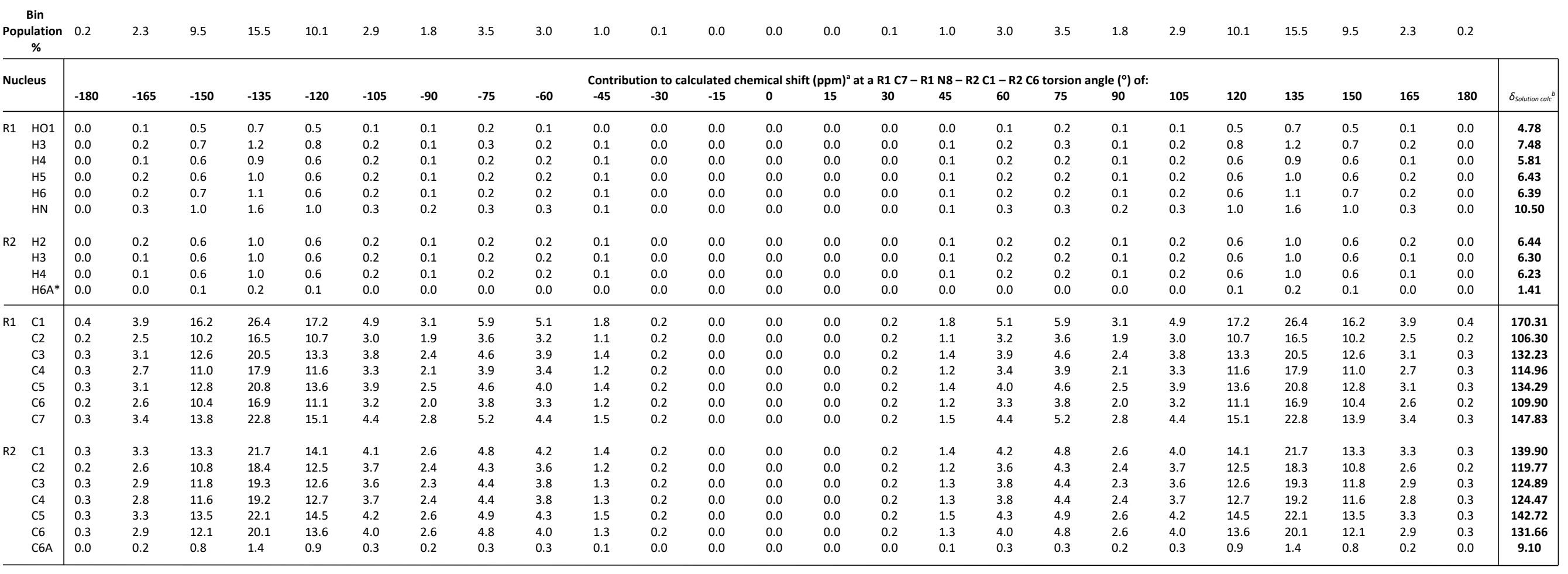

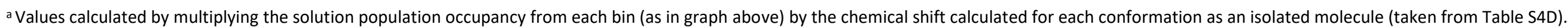

bSum of row (i.e., sum of the individual contributions to the calculated chemical shift from each 15 angle), giving the calculated chemical shift, $\delta_{\text {solution calc. }}$ 
Supplementary Figure $\mathrm{S} 6-(\mathrm{A}, \mathrm{C}){ }^{1} \mathrm{H}(600 \mathrm{MHz}) \mathrm{MAS}(60 \mathrm{kHz})$ and $(\mathrm{B}, \mathrm{D}){ }^{1} \mathrm{H}(500 \mathrm{MHz})-{ }^{13} \mathrm{C} \mathrm{CP}(1 \mathrm{~ms}$ contact time) MAS (12.5 kHz) NMR spectra of ( $A$ and $B$ ) Form I and ( $C$ and $D)$ Form II of TFA. In $B$ and $D$, spinning sidebands are labelled with an asterix. $8(A, C)$ or $1000(B, D)$ transients were co-added. Blue and red labels refer to ring 1 (R1) and 2 (R2) - see Figure 1 in the main text.
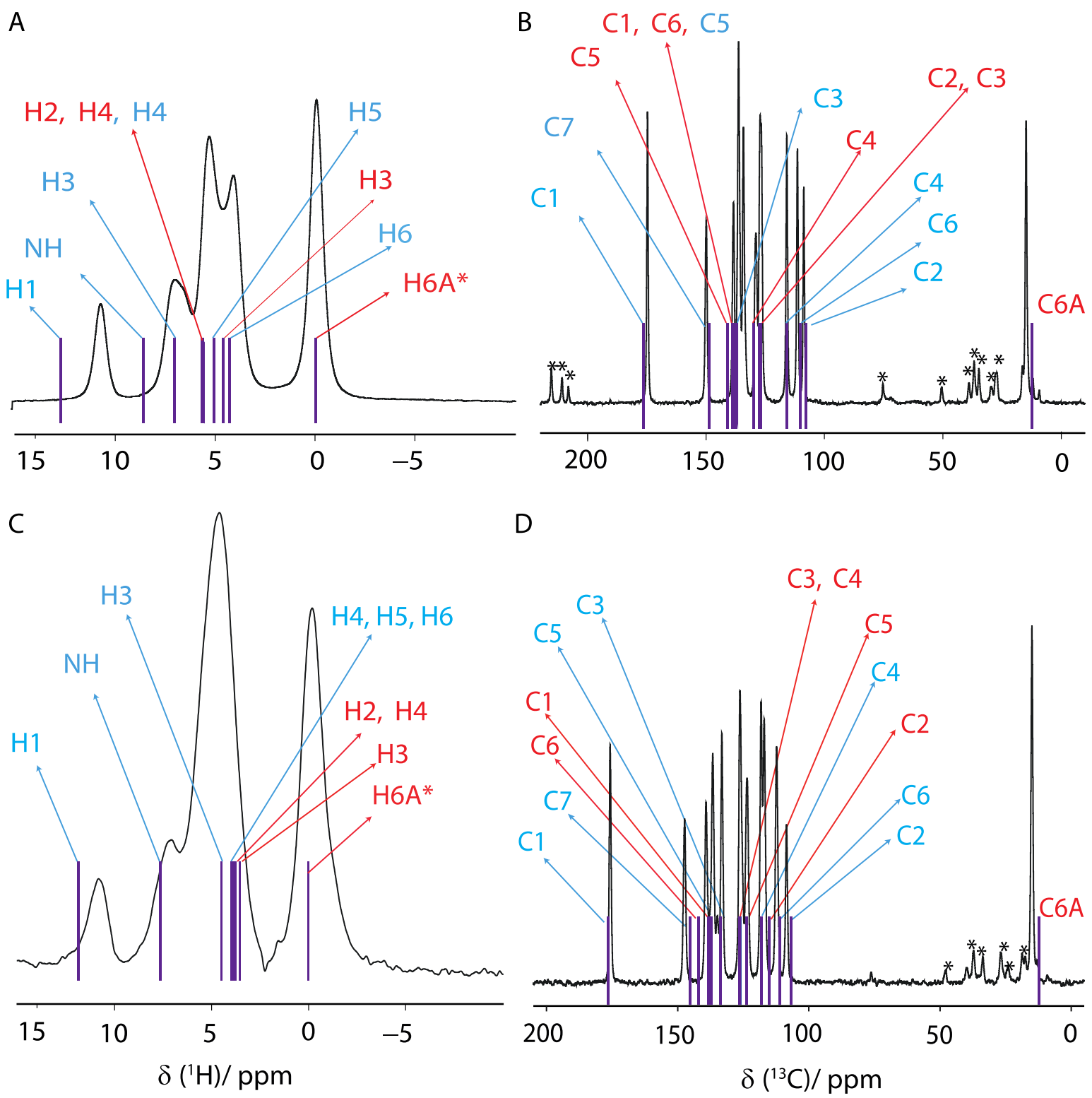
Supplementary Figure $S 7-(A, D) 2 D{ }^{1} \mathrm{H}(600 \mathrm{MHz})(\mathrm{DQ})-{ }^{1} \mathrm{H}(\mathrm{SQ}) \mathrm{MAS}\left(60 \mathrm{kHz}\right.$, with $1 \tau_{\mathrm{R}}$ of BABA recoupling), (B, E) $2 \mathrm{D}^{14} \mathrm{~N}-{ }^{1} \mathrm{H}(600 \mathrm{MHz}) \mathrm{HMQC} \mathrm{MAS}\left(60 \mathrm{kHz}, \tau_{\mathrm{RCPL}}=133 \mu \mathrm{s}\right)$ and $(\mathrm{C}, \mathrm{F}) 2 \mathrm{D}^{1} \mathrm{H}(500 \mathrm{MHz})$ $-{ }^{13} \mathrm{C}$ refocused INEPT (12.5 kHz MAS, spin-echo $(\tau-\pi-\tau)$ duration of $\left.1.44 \mathrm{~ms}\right)$ spectra of Form I $(\mathrm{A}, \mathrm{B}, \mathrm{C}$, left) and Form II (D, E, F, right). The base contour level is at (A) $14 \%$, (B) $2 \%,(C) 37 \%$, (D) $19 \%$, (E) $4 \%$ and (F) $28 \%$ of the maximum peak height. Blue and red labels refer to ring 1 (R1) and 2 (R2) - see Figure 1 in the main text.

\section{Form I}

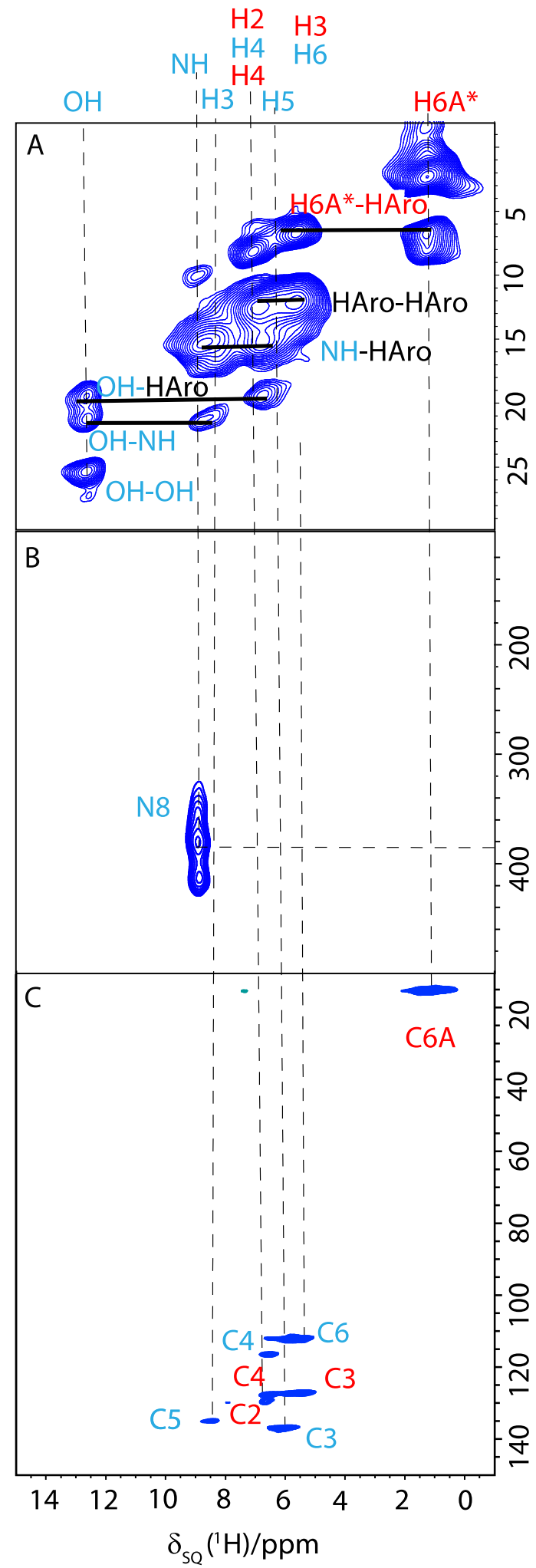

遮

오

우

8

$\infty$

8

은

Q

\section{Form II}

$\mathrm{H} 3 \mathrm{H} 4$

$\mathrm{H} 6 \mathrm{H} 2$

$\mathrm{OH} \quad \mathrm{NHH} 3 \mathrm{H} 4 \mathrm{H} 5$

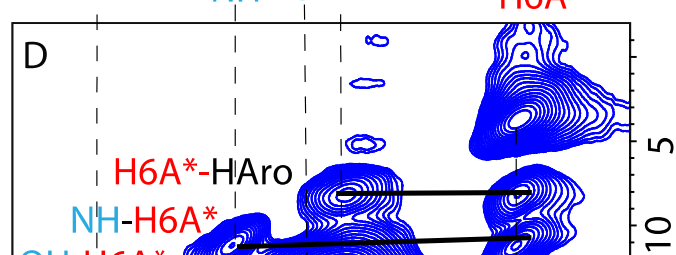

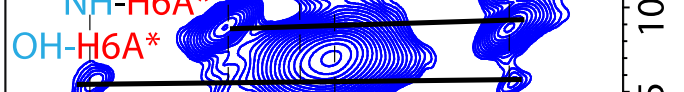
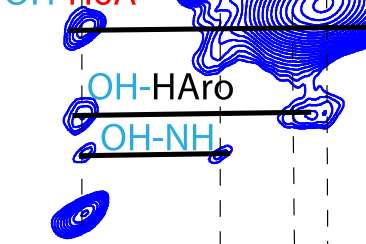

$\mathrm{OH}-\mathrm{OH}$

E

우

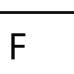

F

$\begin{array}{llllllll}14 & 12 & 10 & 8 & 6 & 4 & 2 & 0\end{array}$

$\delta_{\text {sQ }}\left({ }^{1} \mathrm{H}\right) / \mathrm{ppm}$

우

8

$\infty$

8

ํㅗ

움

$\sum_{\substack{0 \\ 0}}^{\frac{\varepsilon}{2}}$ 


\section{Solid-state NMR Supplementary Figures experimental details}

Experiments were performed at a ${ }^{1} \mathrm{H}$ Larmor frequency of $600 \mathrm{MHz}$ using a $1.3 \mathrm{~mm}$ triple resonance probe (operating in double resonance mode).

${ }^{1} \mathbf{H}-{ }^{1} \mathbf{H}$ Double Quantum (DQ) MAS ${ }^{1}$ : Excitation and reconversion of DQ coherence were achieved using one rotor period of the $\mathrm{BaBa}$ (Back to Back) recoupling sequence ${ }^{2,3}$. A 16-step phase cycle was used to select $\Delta p= \pm 2$ during excitation and $\Delta p=-1$ on the z-filter $90^{\circ}$ pulse, where $p$ is the coherence order. 8 transients were co-added for each of 144 (for Form I) or 160 (for Form II) $t_{1}$ FIDs, using the States method to achieve sign discrimination in $F_{1}$ with a rotor synchronized $t_{1}$ increment of $16.67 \mu \mathrm{s}$, corresponding to a total experimental time of $19 \mathrm{~h}$ (for Form I) or $21 \mathrm{~h}$ (for Form II).

${ }^{14} \mathrm{~N}-{ }^{1} \mathrm{H}$ HMQC experiments: The ${ }^{14} \mathrm{~N}-{ }^{1} \mathrm{H}$ HMQC pulse sequence of $\mathrm{Gan}^{4}$ utilizing rotary resonance recoupling $\left(\mathrm{R}^{3}\right)^{5}$, which applies an ${ }^{1} \mathrm{H} R \mathrm{RF}$ nutation frequency of twice the spinning frequency, was used. A second ${ }^{1} \mathrm{H} 90^{\circ}$ pulse $\left(90^{\circ}\right.$ out of phase with respect to the first $\left.90^{\circ}\right)$ was applied shortly after the first ${ }^{1} \mathrm{H} 90^{\circ}$ pulse, while a phase inversion (every rotor period) of the $\mathrm{R}^{3}$ pulses was used ${ }^{6,7} .8$ transients were co-added for each of $144 t_{1}$ FIDS with a recycle delay of $60 \mathrm{~s}$. A short $R^{3}$ recoupling time of 133.33 $\mu$ s was used to observe one-bond $\mathrm{NH}$ correlations ${ }^{8} .{ }^{14} \mathrm{~N}$ is referenced using the dipeptide $\beta$-AspAla $\left({ }^{14} \mathrm{~N}\right.$ of $\mathrm{NH}_{3}$ peak at $-284 \mathrm{ppm}$ at this magnetic field strength), corresponding to liquid $\mathrm{CH}_{3} \mathrm{NO}_{2}$ at $0 \mathrm{ppm} .{ }^{9}$ 
Supplementary Table S7 - The lower bound of the one-sided $95 \%$ confidence intervals for the correlation of $\Delta \delta_{\text {Experimental }} \mathrm{vs} \Delta \delta_{\text {calculated }}$ and $\delta_{\text {solid expt }} \mathrm{vs} \delta_{\text {solid Calc }}$

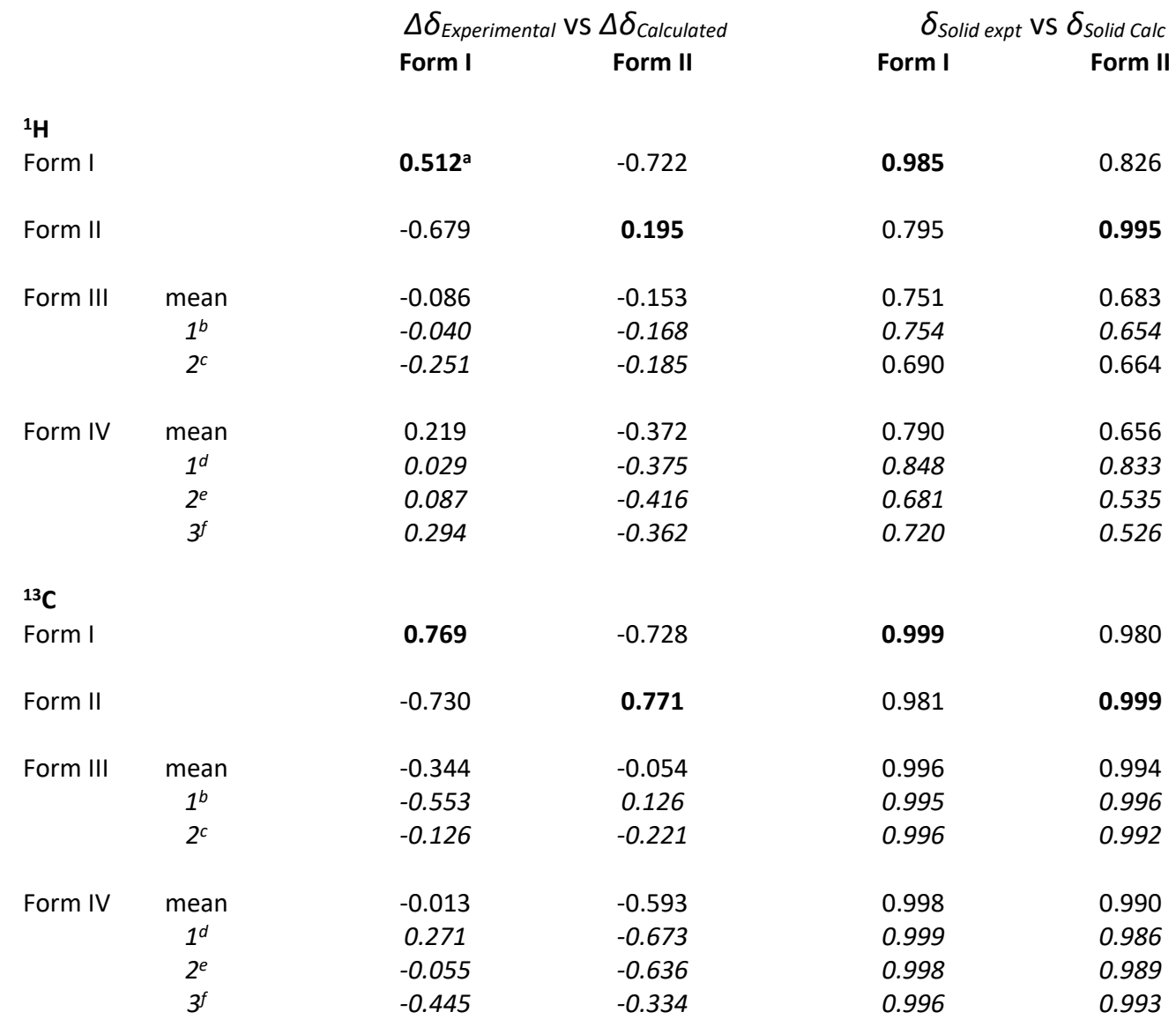

aValues in bold and underlined indicate form corresponding to the measured experimental data, i.e. the ones the approach should identify (see Figures 6, 7, S8 \& S9 and 8, 9, S10 \& S11).

b-f R1 C7 - R1 N8 - R2 C1 - R2 C6 torsion angles are $126.8^{\circ},-138.4^{\circ},-115.8^{\circ},-126.0^{\circ}$ and $-134.2^{\circ}$ in the non DFT geometry optimised crystal structures, respectively. 
Supplementary Figure $\mathbf{S 8}$ - Graphs of $\Delta \delta_{\text {calculated }}$ for each conformation of Form III and Form IV against $\Delta \delta_{\text {Experimental }}$ for Form I. ${ }^{1} \mathrm{H}$ and ${ }^{13} \mathrm{C}$ data shown, with R1 HN and R2 $\mathrm{C} 5$ omitted. See Table 2 for data.

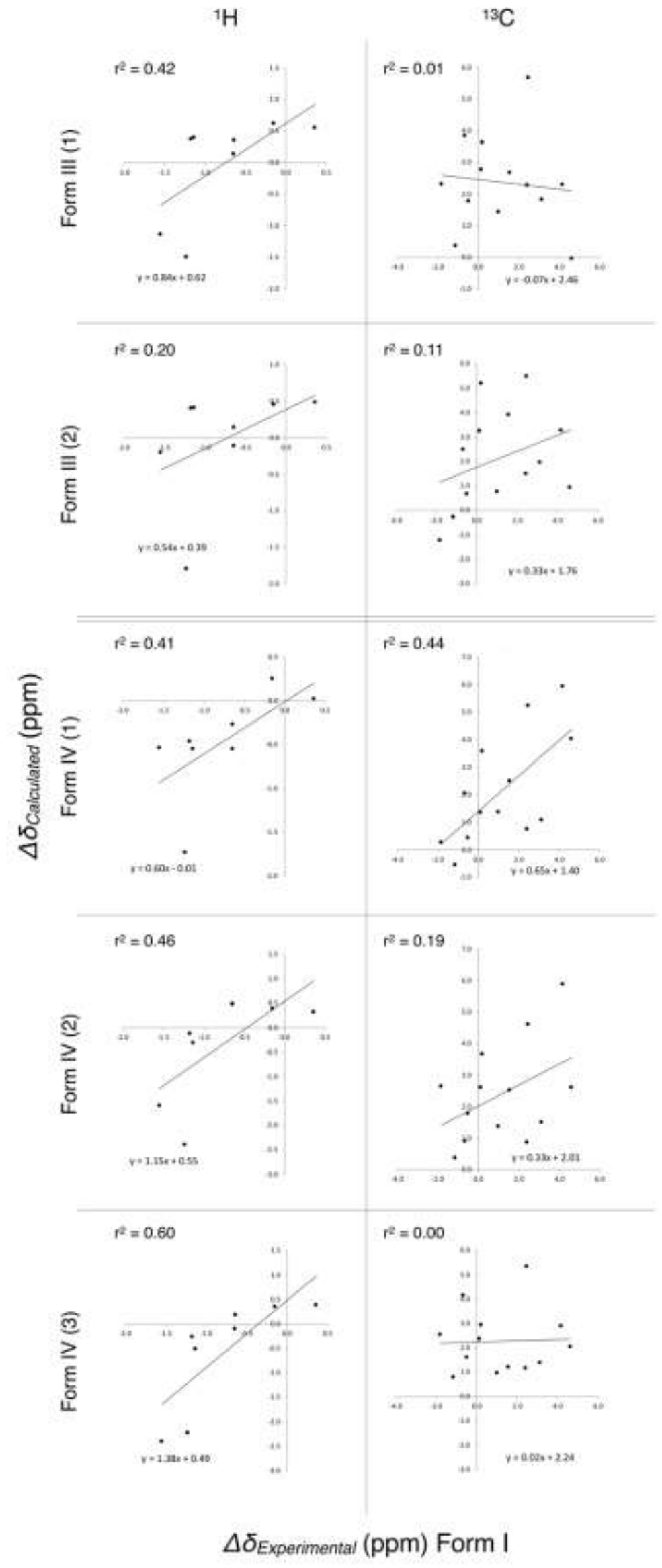


Supplementary Figure $\mathbf{S 9}$ - Graphs of $\Delta \delta_{\text {calculated }}$ for each conformation of Form III and Form IV against $\Delta \delta_{\text {Experimental }}$ for Form II. ${ }^{1} \mathrm{H}$ and ${ }^{13} \mathrm{C}$ data shown, with R1 HN and R2 C5 omitted. See Table 2 for data.

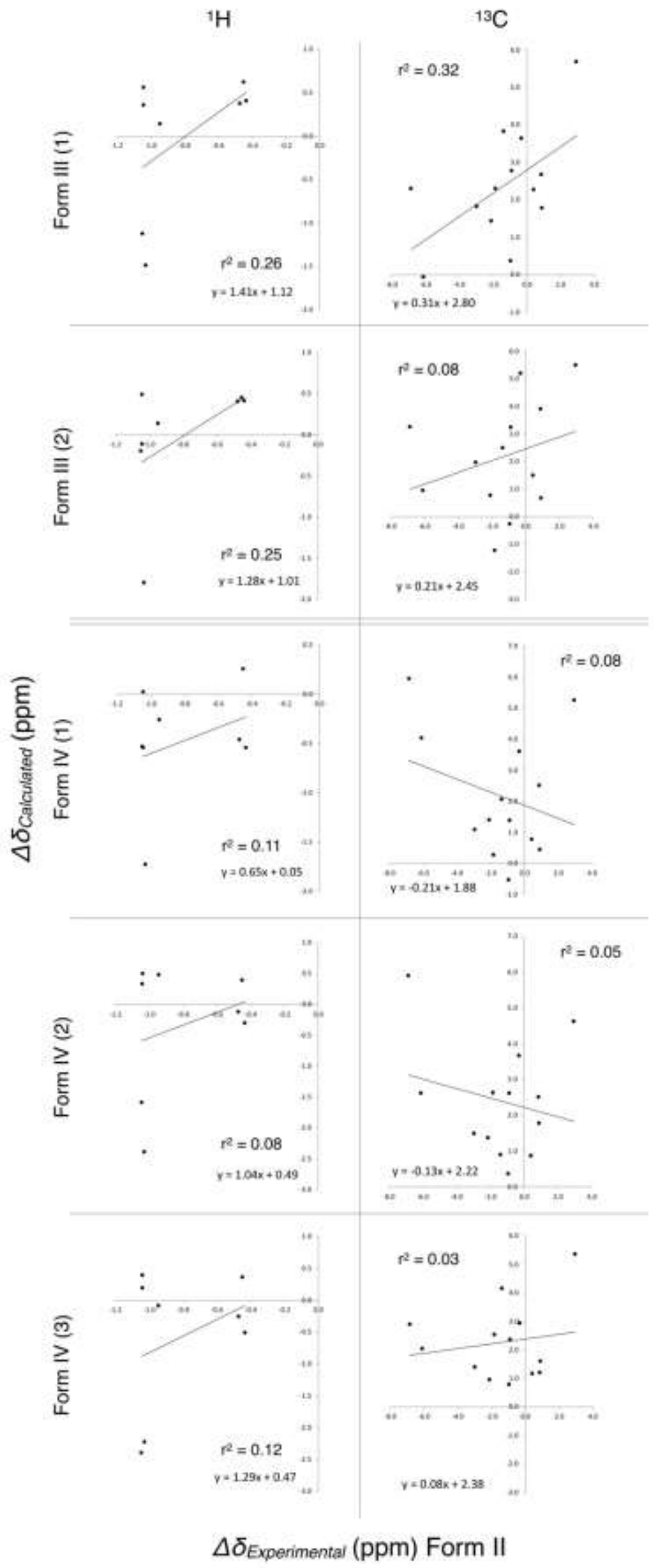


Supplementary Figure $\mathbf{S 1 0}-$ Graphs of $\delta_{\text {solic calc }}$ for each conformation of Form III and Form IV against $\delta_{\text {solid expt }}$ for Form I. ${ }^{1} \mathrm{H}$ and ${ }^{13} \mathrm{C}$ data shown, with R1 HN and R2 C5 omitted. See Table 1 for data.

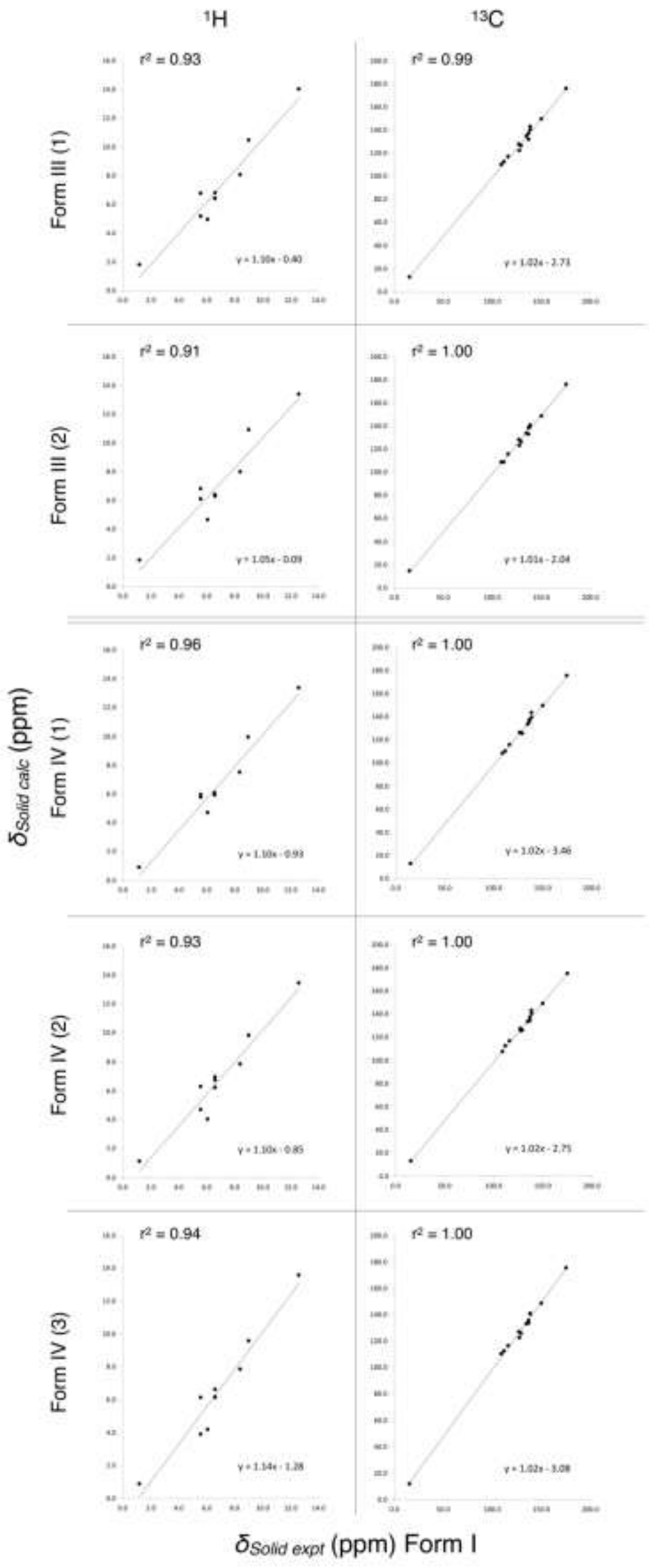


Supplementary Figure S11- Graphs of $\delta_{\text {solic calc }}$ for each conformation of Form III and Form IV against $\delta_{\text {solid expt }}$ for Form II. ${ }^{1} \mathrm{H}$ and ${ }^{13} \mathrm{C}$ data shown, with R1 HN and R2 C5 omitted. See Table 1 for data.

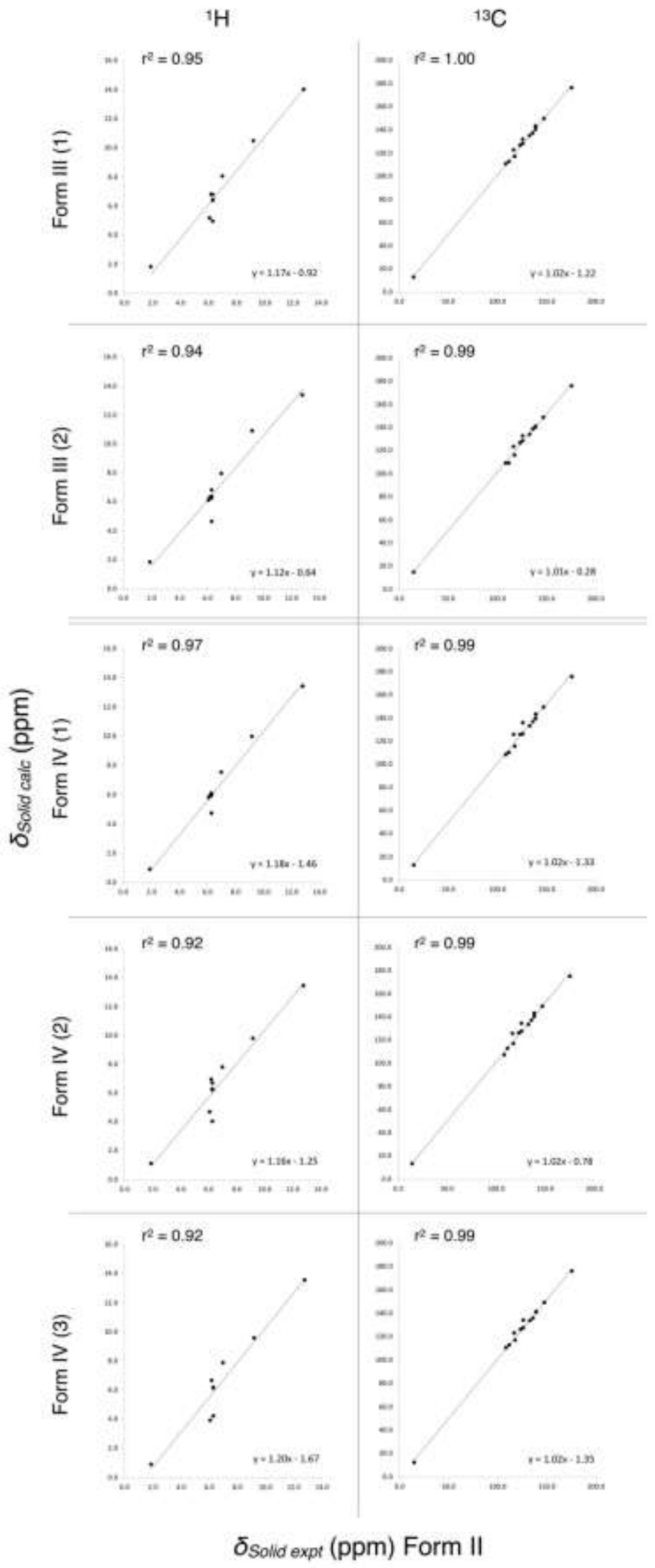


Supplementary Table $\mathbf{8 8}$ - Regression analysis fit parameters (gradient $\mathrm{m}$, intercept $\mathrm{c}$ and coefficient of determination $\mathrm{r}^{2}$ values) and $\mathrm{p}$-values on passing from solution to solid state for combinations of calculated (Form I, II, III and IV) and experimentally measured (Form I and II) changes in chemical shift for a range of solvents $\mathrm{s}^{\mathrm{a}, \mathrm{b}, \mathrm{c}, \mathrm{d}}$

\begin{tabular}{|c|c|c|c|c|c|c|c|c|c|c|c|c|c|c|c|c|c|c|c|c|c|c|c|c|c|c|c|c|c|c|c|c|}
\hline \multirow{2}{*}{ Cilculated chagres tor: } & \multirow{2}{*}{$r^{2}+a r a r$} & \multirow{2}{*}{$\begin{array}{c}\text { Form } 1 \\
\mathrm{~m}\end{array}$} & \multirow{2}{*}{\multicolumn{3}{|c|}{ ONonotorm-d }} & \multirow{2}{*}{$\begin{array}{c}\text { Form } 11 \\
\mathrm{~m}\end{array}$} & \multirow{2}{*}{$c$} & \multirow{2}{*}{$p$} & \multirow{2}{*}{$r^{2}+a+2$} & \multirow{2}{*}{$\begin{array}{l}\text { Ferm } 1 \\
\mathrm{~m}\end{array}$} & \multirow{2}{*}{\multicolumn{3}{|c|}{ Benzzened 16}} & \multirow{2}{*}{$\begin{array}{c}\text { Form II } \\
\mathrm{m}\end{array}$} & \multirow{3}{*}{$\epsilon$} & \multirow{2}{*}{$P$} & \multirow{2}{*}{$r^{2}$} & \multirow[b]{2}{*}{$\begin{array}{c}\text { Form I } \\
\mathrm{m}\end{array}$} & \multirow{2}{*}{\multicolumn{3}{|c|}{ Toluene- ds }} & \multirow{2}{*}{$\underset{\mathrm{m}}{\mathrm{Farm}}$} & \multirow{2}{*}{$c$} & \multirow{2}{*}{$p$} & \multirow{2}{*}{$r$} & \multirow{2}{*}{$\begin{array}{c}\text { Form } 1 \\
\mathrm{~m}\end{array}$} & \multirow{2}{*}{\multicolumn{3}{|c|}{ Pyridne ds }} & \multirow{2}{*}{$\begin{array}{l}\text { Form II } \\
\text { m }\end{array}$} & \multirow{3}{*}{$c$} & \multirow{2}{*}{$P$} \\
\hline & & & & & & & & & & & & & & & & & & & & & & & & & & & & & & & & \\
\hline H & & & & & & & & $r$ & & & 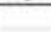 & 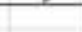 & & & & & & & & & & & & & & & & & & & & \\
\hline Foren ! & 0.74 & 099 & 0.58 & 0.030 & 0.03 & -2.43 & -0.53 & 0.661 & 0.86 & 1.27 & -0.08 & $\leq 0.092$ & a.12 & -0.83 & .028 & 0.802 & 0.85 & 123 & 0.06 & 0.001 & 0.10 & 0.78 & -0.29 & 0.774 & 0.59 & 1.25 & 0.23 & $\{0.001$ & 0.06 & -0.53 & -0.39 & 0.724 \\
\hline Form : & 0.01 & -0.04 & -2.13 & 0586 & 0.54 & 0.75 & 0.30 & 0.019 & 0,00 & -0.02 & -0.10 & 0.533 & 0.69 & 0.82 & -0.01 & 0.005 & 0.00 & -0.00 & -0.10 & 0547 & 0.73 & o89 & 0.02 & 9004 & 0,00 & 000 & -0.10 & 0.504 & 0.70 & 0.78 & 0.19 & 0,000 \\
\hline Form Hif (mean) & 0.33 & 0.69 & 0.51 & 0.069 & 0.27 & 1.35 & 106 & 0.091 & 0.22 & 0.67 & 0.03 & 0.121 & 0.02 & 0.33 & 0.02 & 0.375 & 0.22 & 0.65 & 0.04 & 0.121 & 0.03 & 0.26 & 0.04 & 0.339 & 0.29 & 0.75 & 0.22 & 0.086 & 0.07 & 0.62 & a.21 & 0.251 \\
\hline $\begin{array}{l}\mathrm{C} c \\
\text { ferm! }\end{array}$ & 0.83 & & & & & & & & & & 105 & & & & & & 0.87 & & & & 0.11 & & & & $a n$ & & 187 & & 000 & & & \\
\hline 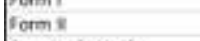 & 0.15 & -0.62 & 0.98 & 0.905 & 0.23 & 1.09 & 170 & $\leq 0.001$ & 0.11 & $\begin{array}{l}-0.52 \\
-52-10\end{array}$ & 11) & 0.860 & as? & 1.10 & 1.16 & s0001 & 0.12 & -0.52 & 1.18 & 0.872 & 0.87 & 1.11 & 1.12 & $\$ 0.001$ & 0.11 & .0 .932 & 0.85 & 0.857 & 0.62 & 0.95 & a.9a & $\leq 0.013$ \\
\hline Form wimesn) & 0.03 & $a 13$ & 2.11 & 0.301 & 0.19 & 0.26 & 263 & 0.069 & 0.05 & 0.17 & 197 & 0.242 & 0.22 & 0.28 & 2.49 & 0.052 & 0.04 & 0.15 & 1.98 & 0.249 & 0.23 & 0.29 & 2.49 & 0.050 & 0.01 & 0.05 & 217 & 0.364 & 0.20 & 0.27 & 246 & 0.051 \\
\hline Form $N$ imean] & 0.22 & 0.33 & 1.89 & 0.053 & 0.03 & -009 & 216 & 0.700 & 0.25 & 0.35 & 166 & 0.040 & $\alpha_{0.2}$ & -0.07 & 2.23 & 0.657 & 0.25 & 0.34 & 1.65 & 0.040 & 0.01 & -0.06 & 2.23 & 0.645 & 0.19 & 0.19 & 194 & 0.069 & 000 & 0.92 & 2.30 & 0.458 \\
\hline
\end{tabular}

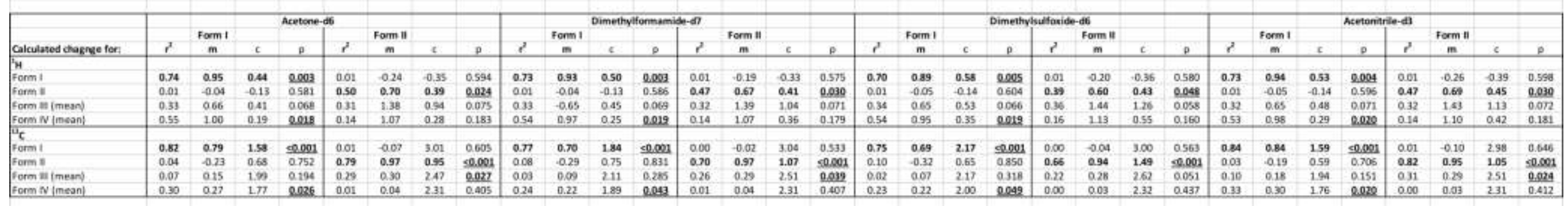

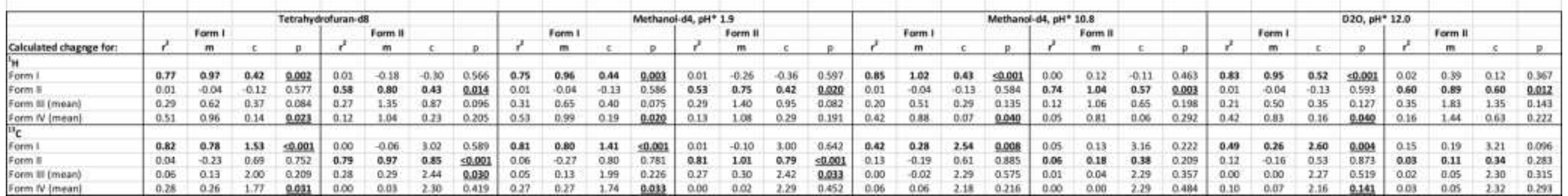

${ }^{a}$ Chemical shift data for the fits were taken from Crick et al. ${ }^{54}$

${ }^{b}$ Values in bold indicate the fit parameters for the form corresponding to the measured experimental data, i.e., the ones the approach should identify.

${ }^{\mathrm{c}} \mathrm{pH}^{*}$ indicates the observed $\mathrm{pH}$ value, uncorrected for solvent composition.

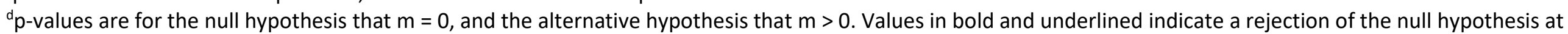

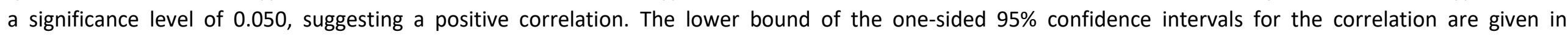
supplementary Table S9. 
Supplementary Table S9 - The lower bound of the one-sided $95 \%$ confidence intervals for the correlation of $\Delta \delta_{\text {Experimental }}$ Vs $\Delta \delta_{\text {calculated }}$ and $\delta_{\text {solid expt }}$ Vs $\delta_{\text {solid Calc }}$ for a range of solvents ${ }^{\mathrm{a}, \mathrm{b}}$

\begin{tabular}{|c|c|c|c|c|c|c|c|c|}
\hline & Chloroform-d & & Benzene-d6 & & Toluene- $d 8$ & & Pyridine-d5 & \\
\hline & Form I & Form II & Form I & Form II & \begin{tabular}{|l} 
Form I \\
\end{tabular} & Form I I & Form I & Form II \\
\hline \multicolumn{9}{|l|}{${ }^{1} \mathrm{H}$} \\
\hline Form I & 0.512 & -0.772 & 0.713 & -0.800 & 0.707 & -0.785 & 0.770 & -0.759 \\
\hline Form II & -0.679 & 0.195 & -0.086 & 0.431 & -0.656 & 0.483 & -0.629 & Q.446 \\
\hline Form III (mean) & -0.086 & -0.153 & -0.294 & -0.537 & -0.301 & -0.507 & -0.137 & -0.432 \\
\hline Form IV (mean) & 0.219 & -0.372 & 0.093 & -0.650 & 0.095 & -0.623 & 0.196 & -0.570 \\
\hline \multicolumn{9}{|l|}{${ }^{13} \mathrm{c}$} \\
\hline Form I & 0.769 & -0.728 & 0.813 & -0.709 & 0.817 & -0.700 & 0.621 & -0.484 \\
\hline form II & -0.730 & 0.771 & -0.701 & 0.816 & -0.704 & 0.822 & -0.702 & 0.497 \\
\hline Form III (mean) & -0.344 & -0.054 & -0.224 & -0.008 & -0.223 & -0.001 & -0.391 & -0.033 \\
\hline Form IV (mean) & -0.013 & -0.593 & 0.033 & -0.568 & 0.034 & -0.562 & -0.054 & -0.452 \\
\hline
\end{tabular}

\begin{tabular}{|c|c|c|c|c|c|c|c|c|}
\hline & \multirow{2}{*}{$\begin{array}{c}\text { Acetone-d } 6 \\
\text { Form I }\end{array}$} & \multirow[b]{2}{*}{ Form II } & \multicolumn{2}{|c|}{ Dimethylformamide-d7 } & \multicolumn{2}{|c|}{ Dimethyisulfoxide-d6 } & \multicolumn{2}{|c|}{ Acetonitrile-d3 } \\
\hline & & & Form I & Form II & Form I & Form II & Form I & Form 1 \\
\hline \multicolumn{9}{|l|}{ ['H } \\
\hline Form I & 0.510 & -0.684 & 0.498 & $-0,672$ & 0.438 & -0.676 & 0.487 & -0.687 \\
\hline Form II & -0.676 & 0.150 & -0.679 & 0.104 & -0.690 & 0.001 & -0.686 & e.104 \\
\hline Form III (mean) & -0.247 & -0.106 & -0.083 & -0.091 & -0.072 & -0.043 & -0.091 & -0.094 \\
\hline Form IV (mean) & 0.210 & -0.333 & 0.197 & -0.326 & 0.197 & -0.297 & 0.192 & -0.331 \\
\hline \multicolumn{9}{|l|}{${ }^{13} \mathrm{C}$} \\
\hline Form I & 0.760 & -0.539 & 0.692 & -0.497 & 2,660 & -0.514 & 0.776 & -0.562 \\
\hline Form II & -0.624 & 0.718 & -0.673 & 0.592 & -0.687 & 0.547 & -0.596 & 0.756 \\
\hline Form III (mean) & -0.082 & 0.087 & -0.331 & 0.036 & -0.357 & -0.007 & -0.196 & 0.106 \\
\hline Form IV (mean) & 0.099 & -0.418 & 0.023 & -0.420 & 0.000 & -0.439 & 0.136 & -0.423 \\
\hline
\end{tabular}

\begin{tabular}{|c|c|c|c|c|c|c|c|c|}
\hline & \multicolumn{2}{|c|}{ Tetrahydrofuran-d8 } & \multicolumn{2}{|c|}{ Methanol-d4, $\mathrm{pH}^{*} 1.9$} & \multicolumn{2}{|c|}{ Methanol-d4, $\mathrm{pH}^{*} 10.8$} & \multicolumn{2}{|c|}{$\mathrm{D} 2 \mathrm{O}, \mathrm{pH}^{*} 12.0$} \\
\hline & Form 1 & Form II & Form I & Form II & Form I & Form II & Form I & Form II \\
\hline \multicolumn{9}{|l|}{${ }^{3} \mathrm{H}$} \\
\hline Form I & 0.556 & -0.668 & 0.522 & -0.686 & 0.694 & -0.602 & 0.656 & 0.355 \\
\hline Form II & -0.674 & 0.260 & -0.679 & 0.189 & -0.678 & 0.511 & -0.683 & 0.290 \\
\hline Form III (mean) & -0.131 & -0.165 & -0.104 & -0.127 & -0.252 & -0.355 & -0.489 & -0.267 \\
\hline Form IV (mean) & 0.161 & -0.363 & 0.191 & -0.344 & 0.042 & -0.463 & 0.043 & -0.386 \\
\hline \multicolumn{9}{|l|}{${ }^{13} \mathrm{C}$} \\
\hline Form 1 & 0.754 & -0.529 & 0.742 & -0.560 & Q.253 & -0.275 & 0.337 & -0.112 \\
\hline Form II & -0.623 & 0.720 & -0.641 & 0.735 & -0.714 & -0.262 & -0.704 & -0.330 \\
\hline Form ill (mean) & -0.263 & 0.075 & -0.280 & 0.061 & .0 .522 & .0 .386 & -0.236 & -0.355 \\
\hline Form IV (mean) & 0.069 & -0.428 & 0.060 & -0.448 & -0.270 & -0.468 & -0.183 & -0.338 \\
\hline
\end{tabular}

${ }^{a}$ Values in bold and underlined indicate form corresponding to the measured experimental data, i.e. the ones the approach should identify. ${ }^{\mathrm{b}} \mathrm{pH}^{*}$ indicates the observed $\mathrm{pH}$ value, uncorrected for solvent composition. 
Supplementary Figure S12 - Comparison of conformations from each crystal form with the dynamic 3D structure of TFA. Form I adopts conformations within the minor R1 C7 - R1 N8 - R2 C1 - R2 +/- 70 mode, whereas Forms II, III and IV all adopt conformations within the major $+/-135^{\circ}$ mode (note that there are two and three distinct TFA molecules in Forms III and IV, respectively). The extent of libration is illustrated by the shadows.
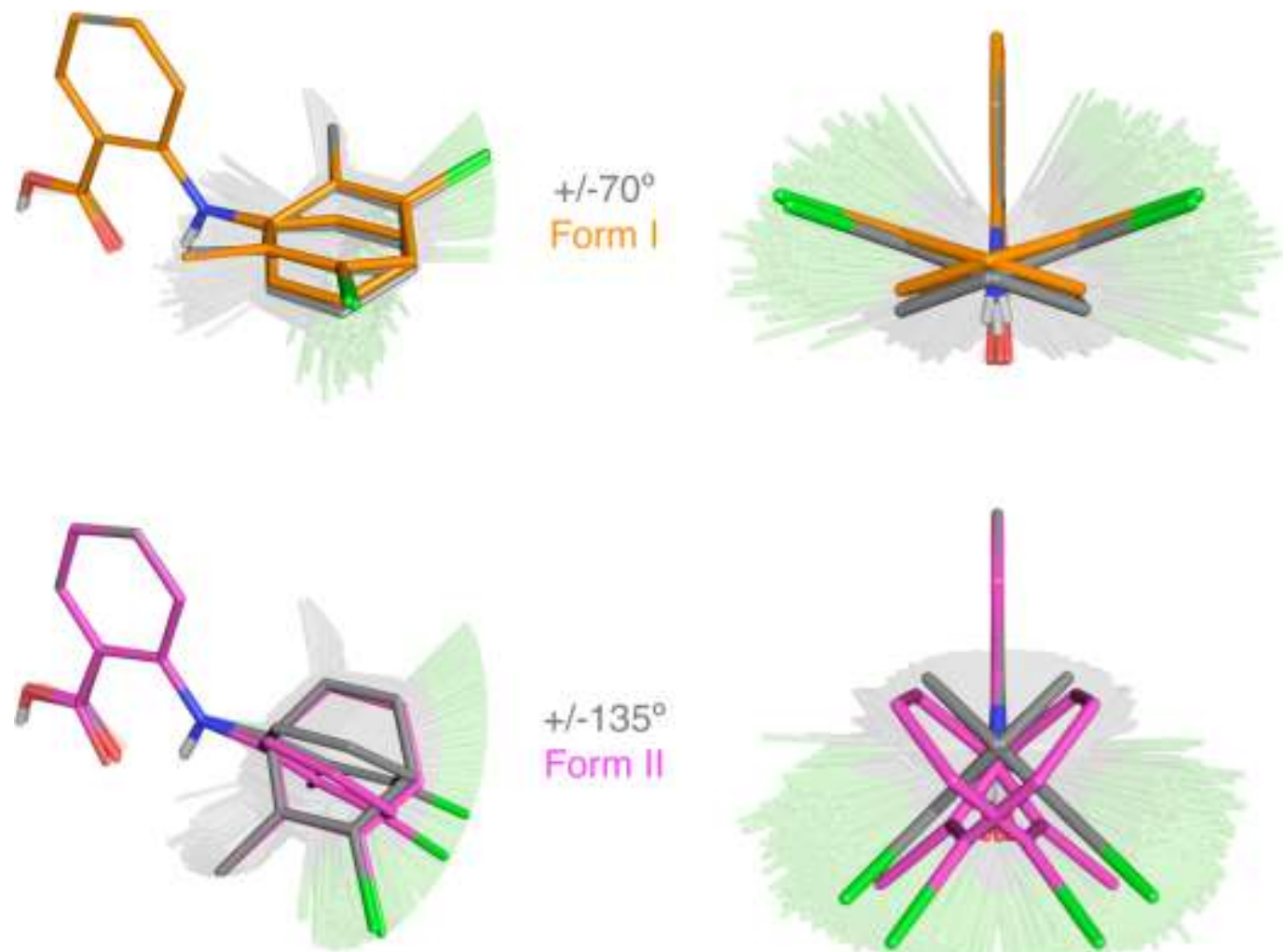

$+/-135^{\circ}$

Form II
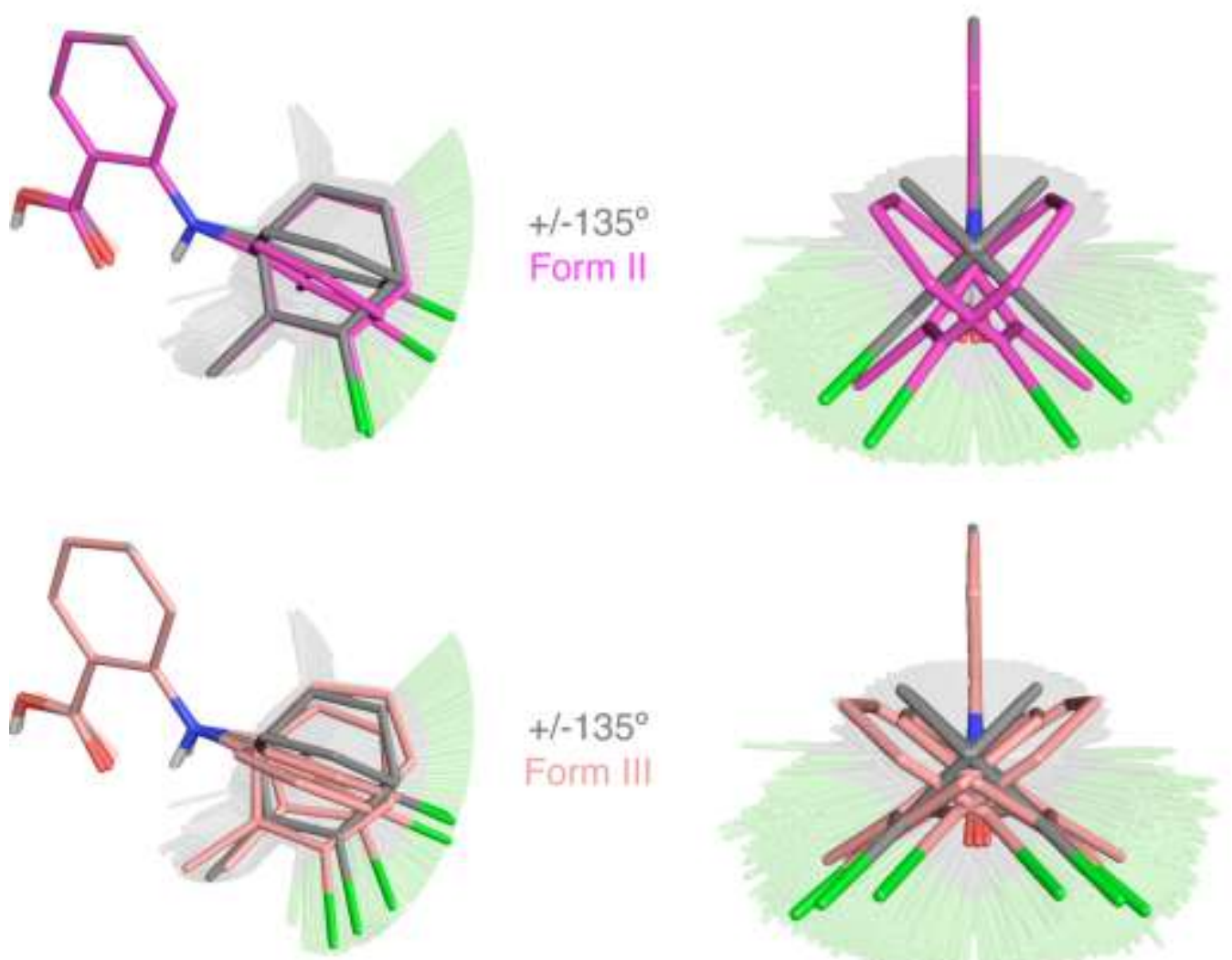

$+/-135^{\circ}$

Form III
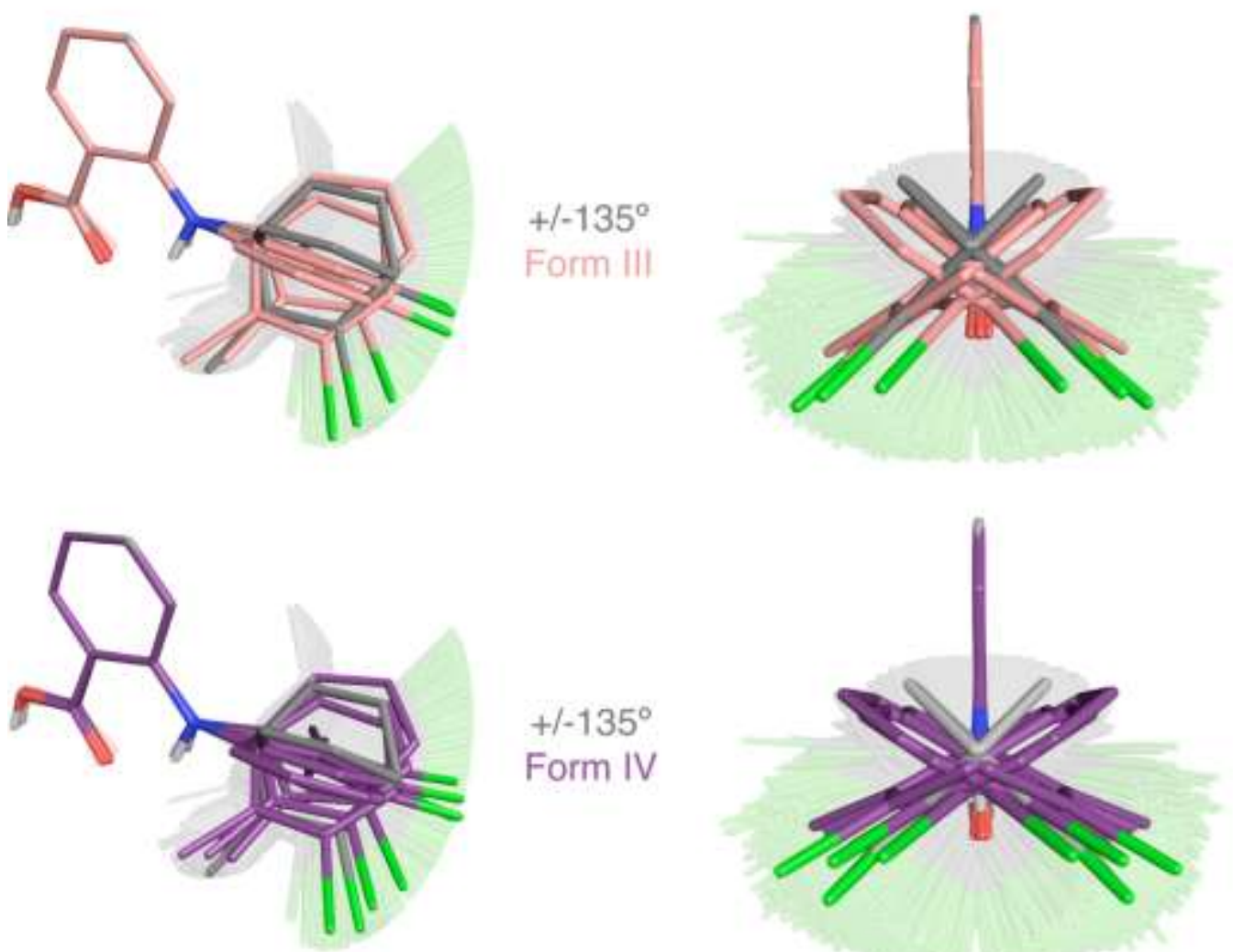

$+/-135^{\circ}$

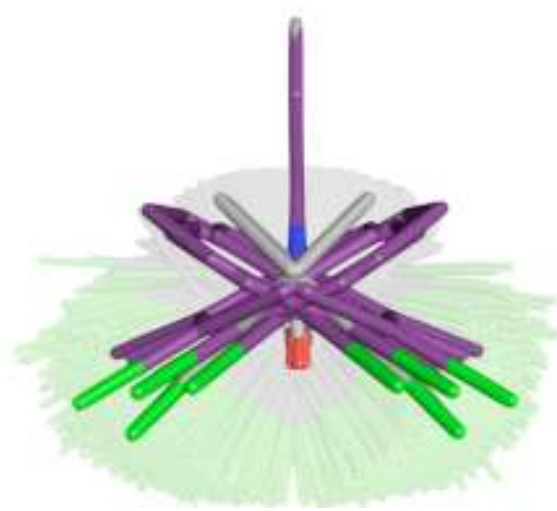


Supplementary Figure S13 - Continuous line graph representing the solution conformational distribution for TFA compared to conformations found in crystal forms, for torsion R1 C7 - R1 N8 - R2 $\mathrm{C} 1$ - R2. Torsion angles found in the Forms I, II, II and IV $\left(Z^{\prime}=1,1,2\right.$ and 3, respectively) crystal structures (CSD accession/ref codes 1960856, 1960855, KAXXAI02 and KAXXAI03, respectively) are indicated with arrows; note that these are close to, but not coincident with, the solution maxima. Values are Form I: $+/-74.9^{\circ}$, Form II: +/- 142. $6^{\circ}$, Form III: $+/-126.8^{\circ}, 138.4^{\circ}$, Form IV: +/- $115.8^{\circ}, 126.0^{\circ}$, $134.2^{\circ}$.
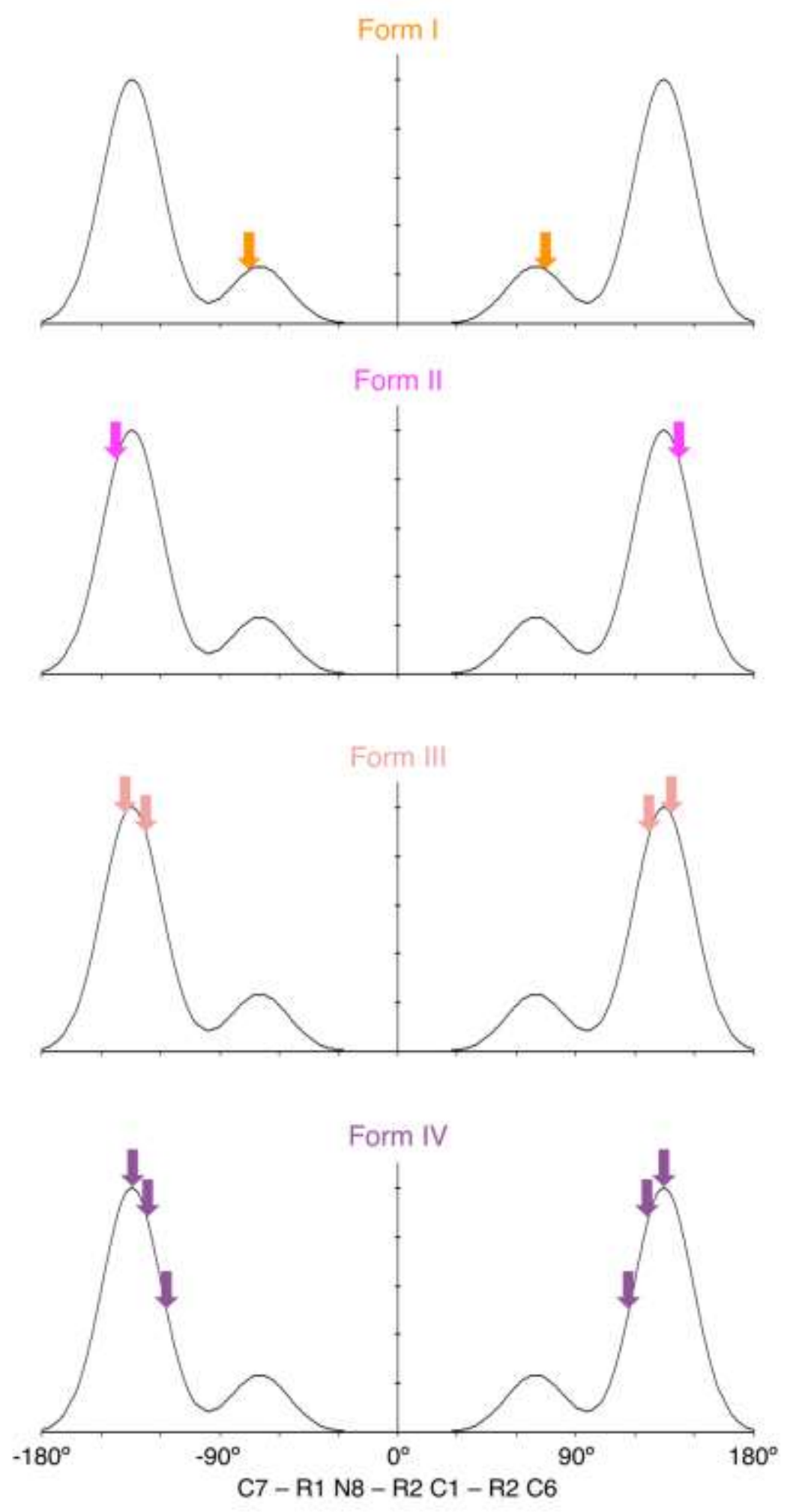
Supplementary Table S10 - Comparison of experimentally observed solution and crystal conformations for torsion R1 C7 - R1 N8 - R2 C1 - R2

$\begin{array}{lccc}\text { State } & \text { Conformation } & \begin{array}{c}\text { Occupancy } \\ \text { \% }^{\mathbf{a}}\end{array} & \mathbf{R 1 ~ C 7 ~ - ~ R 1 ~ N 8 ~ - ~ R 2 ~ C 1 ~ - ~ R ~} \\ \text { Solution } & 1 & 40.5 & \\ & 2 & 40.5 & 134.5 \pm 15^{\mathrm{b}} \\ & 3 & 9.5 & -134.5 \pm 15 \\ & 4 & 9.5 & 69.8 \pm 15 \\ \text { Form I } & 1 & 50 & -69.8 \pm 15 \\ \text { (1960856) } & 2 & 50 & 74.9 \\ & & & -74.9 \\ \text { Form II } & 1 & 50 & 142.6 \\ \text { (1960855) } & 2 & 50 & -142.6 \\ & & & \\ \text { Form III } & 1 & 25 & 126.8 \\ \text { (KAXXAI02) } & 2 & 25 & -126.8 \\ & 3 & 25 & 138.4 \\ & 4 & 25 & -138.4 \\ \text { Form IV } & & & \\ \text { (KAXXAI03) } & 1 & 16.7 & -115.8 \\ & 2 & 16.7 & 126.0 \\ & 3 & 16.7 & -126.0 \\ & 4 & 16.7 & 134.2 \\ & 5 & 16.7 & -134.2\end{array}$

${ }^{a}$ Occupancy as a \% of the total observed in each state.

${ }^{b} \pm$ value represents solution libration about each mean. 
Supplementary Table S11 - Crystal packing similarity between TFA forms, as calculated using the crystal packing similarity tool within Mercury using default settings

\begin{tabular}{lcccc} 
& $\begin{array}{c}\text { Form I } \\
(\mathbf{1 9 6 0 8 5 6 )}\end{array}$ & $\begin{array}{c}\text { Form II } \\
(\mathbf{1 9 6 0 8 5 5 )}\end{array}$ & $\begin{array}{c}\text { Form III } \\
\text { KAXXAI02 }\end{array}$ & $\begin{array}{c}\text { Form IV } \\
\text { KAXXAI03 }\end{array}$ \\
$\begin{array}{l}\text { Form I } \\
(\mathbf{1 9 6 0 8 5 6 )}\end{array}$ & - & $2(1.055)^{\mathrm{a}}$ & $2(0.768)$ & $2(0.685)$ \\
$\begin{array}{l}\text { Form II } \\
(\mathbf{1 9 6 0 8 5 5 )}\end{array}$ & $2(1.055)$ & - & $2(0.281)$ & $2(0.319)$ \\
$\begin{array}{l}\text { Form III } \\
\text { (KAXXAI02) }\end{array}$ & $2(0.768)$ & $2(0.281)$ & & $11(0.479)$ \\
$\begin{array}{l}\text { Form IV } \\
\text { (KAXXAI03) }\end{array}$ & $2(0.685)$ & $2(0.319)$ & $11(0.479)$ & \\
\hline
\end{tabular}

a Number of molecules in common and RMS out of 15. 
Supplementary Table S12 - Calculated chemical shifts for each crystal form conformation as an isolated molecule in an enlarged unit cell

\begin{tabular}{|c|c|c|c|c|c|c|c|c|}
\hline & & & $\delta_{\text {Isola }}$ & pm) & & & & \\
\hline & & $\begin{array}{c}\text { Form I } \\
(1960856)\end{array}$ & $\begin{array}{c}\text { Form II } \\
(1960855)\end{array}$ & $\begin{array}{r}F c \\
\text { (KA }\end{array}$ & $\begin{array}{l}n \text { III } \\
\text { (AIO2) }\end{array}$ & & $\begin{array}{l}\text { Form I } \\
\text { AXXAI }\end{array}$ & \\
\hline & & & & $1^{\mathrm{a}}$ & $2^{b}$ & $1^{c}$ & $2^{d}$ & $3^{\mathbf{e}}$ \\
\hline R1 & $\mathrm{H} 1$ & 6.1 & 6.2 & 6.2 & 6.2 & 6.2 & 6.2 & 6.3 \\
\hline & H3 & 7.3 & 7.4 & 7.3 & 7.4 & 7.4 & 7.3 & 7.4 \\
\hline & $\mathrm{H} 4$ & 5.7 & 5.7 & 5.8 & 5.8 & 5.7 & 5.7 & 5.7 \\
\hline & H5 & 6.2 & 6.3 & 6.4 & 6.3 & 6.4 & 6.3 & 6.3 \\
\hline & H6 & 5.2 & 6.7 & 6.6 & 6.5 & 6.2 & 6.1 & 6.3 \\
\hline & $\mathrm{HN}$ & 9.9 & 10.8 & 11.1 & 10.4 & 10.6 & 10.2 & 9.9 \\
\hline R2 & $\mathrm{H} 2$ & 6.0 & 6.5 & 6.4 & 6.5 & 6.4 & 6.4 & 6.4 \\
\hline & H3 & 6.4 & 6.1 & 6.3 & 6.2 & 6.3 & 6.3 & 6.2 \\
\hline & $\mathrm{H} 4$ & 6.3 & 6.1 & 6.2 & 6.2 & 6.3 & 6.2 & 6.2 \\
\hline & $H 6 A^{* f}$ & 1.1 & 1.4 & 1.4 & 1.4 & 1.3 & 1.4 & 1.4 \\
\hline & H6A1 & 0.9 & 1.1 & 1.1 & 1.0 & 1.1 & 1.0 & 0.9 \\
\hline & H6A2 & 1.0 & 1.6 & 1.2 & 1.5 & 1.3 & 1.5 & 1.5 \\
\hline & H6A3 & 1.3 & 1.6 & 2.0 & 1.7 & 1.6 & 1.6 & 1.7 \\
\hline R1 & C1 & 171.4 & 172.0 & 171.8 & 171.4 & 171.6 & 171.4 & 171.0 \\
\hline & C2 & 104.7 & 106.2 & 105.3 & 106.7 & 105.2 & 105.5 & 107.3 \\
\hline & C3 & 130.8 & 131.3 & 131.0 & 131.3 & 131.2 & 131.1 & 131.2 \\
\hline & C4 & 113.7 & 114.0 & 114.6 & 115.0 & 114.2 & 114.1 & 115.1 \\
\hline & C5 & 133.6 & 133.4 & 134.1 & 133.5 & 134.0 & 133.5 & 133.2 \\
\hline & C6 & 111.1 & 109.5 & 108.3 & 111.6 & 108.8 & 110.2 & 112.3 \\
\hline & $\mathrm{C7}$ & 149.0 & 145.5 & 146.7 & 147.4 & 148.3 & 148.3 & 148.0 \\
\hline R2 & $\mathrm{C} 1$ & 139.5 & 138.8 & 139.6 & 140.0 & 139.2 & 139.7 & 140.4 \\
\hline & C2 & 124.5 & 115.1 & 119.1 & 119.0 & 123.7 & 123.0 & 121.0 \\
\hline & C3 & 126.5 & 124.5 & 124.6 & 124.3 & 125.0 & 125.2 & 124.8 \\
\hline & C4 & 126.7 & 122.1 & 123.4 & 123.9 & 124.8 & 124.9 & 124.3 \\
\hline & C5 & 142.6 & 141.9 & 140.8 & 141.3 & 142.9 & 142.3 & 141.5 \\
\hline & C6 & 137.5 & 128.6 & 130.9 & 131.0 & 134.0 & 133.8 & 131.9 \\
\hline & C6A & 9.0 & 8.6 & 9.2 & 8.1 & 9.3 & 8.7 & 7.5 \\
\hline
\end{tabular}

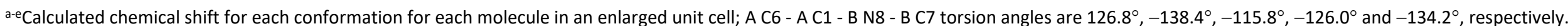
${ }^{f}$ Mean of chemical shifts for all three hydrogen nuclei for comparison with experimental data. 
Supplementary Figure S14 - Plots of $\Delta \delta_{\text {calculated-Isolated }}$ for Forms I, II, III and IV against $\Delta \delta_{\text {Experimental }}$ for Form I for both ${ }^{1} \mathrm{H}$ and ${ }^{13} \mathrm{C}$ showing that the approach still discriminates Form I on ${ }^{13} \mathrm{C}\left(\mathrm{r}^{2}=0.46, p=\right.$ $0.006)$ even after packing effects have been removed. See Table 2 and supplementary Table S12 for data.

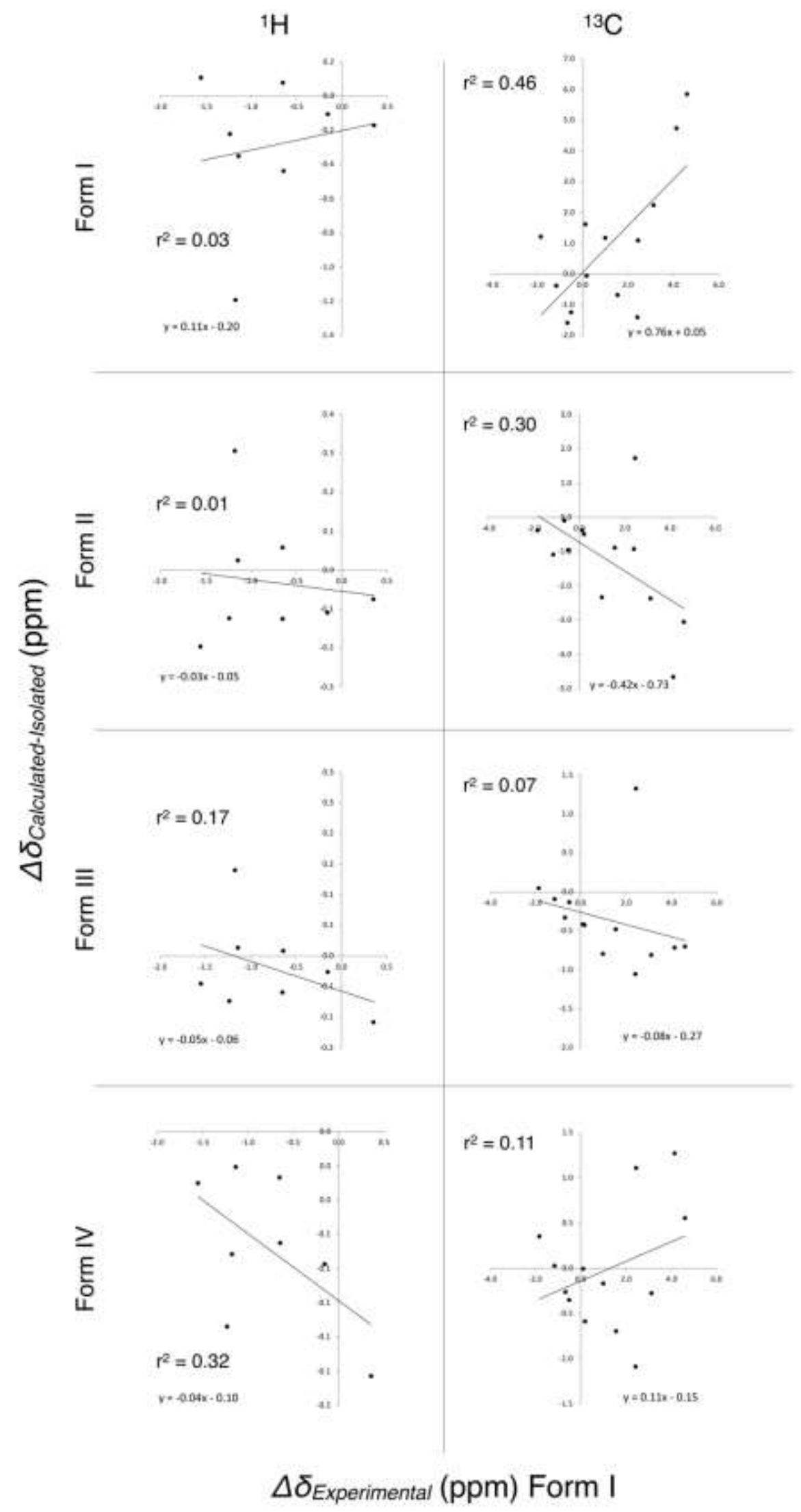


Supplementary Figure S15 - Plots of $\Delta \delta_{\text {calculated-Isolated }}$ for Forms I, II, III and IV against $\Delta \delta_{\text {Experimental }}$ for Form II for both ${ }^{1} \mathrm{H}$ and ${ }^{13} \mathrm{C}$ showing that the approach still discriminates Form II on ${ }^{13} \mathrm{C}\left(\mathrm{r}^{2}=\right.$ positive correlation of $0.78, p<0.001$ ) even after packing effects have been removed. See Table 2 and supplementary Table S12 for data.

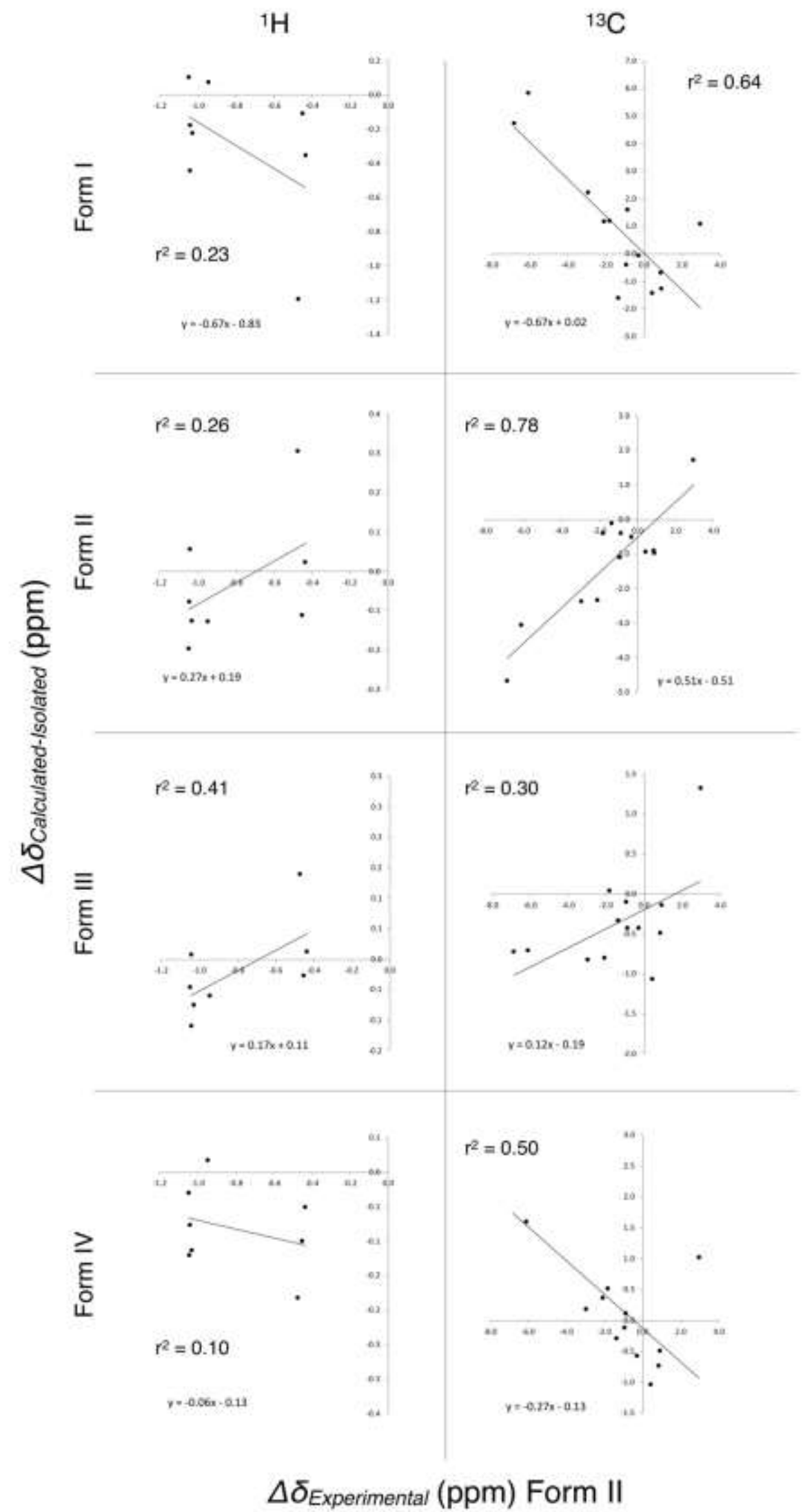


Supplementary Table S13 - The lower bound of the one-sided 95\% confidence intervals for the correlation of $\Delta \delta_{\text {Experimental }}$ Vs $\Delta \delta_{\text {calculated-lsolated. }}$

\begin{tabular}{|c|c|c|c|}
\hline & & $\Delta \delta_{\text {Exper }}$ & $\Delta \delta_{\text {Calculat }}$ \\
\hline & & Form I & Form II \\
\hline${ }^{1} \mathrm{H}$ & & & \\
\hline Form I & & $-0.508^{a}$ & -0.852 \\
\hline Form II & & -0.694 & -0.172 \\
\hline Form III & mean & -0.823 & 0.033 \\
\hline & $1^{b}$ & -0.900 & -0.034 \\
\hline & $2^{c}$ & -0.660 & -0.074 \\
\hline Form IV & mean & -0.705 & -0.419 \\
\hline & $1^{d}$ & -0.703 & -0.784 \\
\hline & $2^{e}$ & -0.754 & -0.874 \\
\hline & $3^{f}$ & -0.541 & -0.445 \\
\hline${ }^{13} \mathrm{C}$ & & & \\
\hline Form I & & 0.294 & -0.924 \\
\hline Form II & & -0.812 & 0.702 \\
\hline Form III & mean & -0.661 & 0.095 \\
\hline & $1^{b}$ & -0.396 & 0.060 \\
\hline & $2^{c}$ & -0.783 & -0.216 \\
\hline Form IV & mean & -0.178 & -0.746 \\
\hline & $1^{d}$ & 0.102 & -0.886 \\
\hline & $2^{e}$ & 0.475 & -0.860 \\
\hline & $3^{f}$ & -0.215 & -0.691 \\
\hline
\end{tabular}

aValues in bold and underlined indicate form corresponding to the measured experimental data, i.e. the ones the approach should identify (see Figures S14 \& S15).

b-fR1 C7 - R1 N8 - R2 C1 - R2 C6 torsion angles are $126.8^{\circ},-138.4^{\circ},-115.8^{\circ},-126.0^{\circ}$ and $-134.2^{\circ}$ in the non DFT geometry optimised crystal structures, respectively. 
Supplementary Figure S16 - Regression analysis parameters (gradient $\mathrm{m}$ and coefficient of determination $\mathrm{r}^{2}$ ) for plots of $\Delta \delta_{\text {calculated-lsolated conformation at specific } 15^{\circ} \text { angle }}$ (i.e., replacing $\delta_{\text {solid calc }}$ by $\delta_{\text {Isolated }}$ conformation at specific $15^{\circ}$ angle for each $15^{\circ}$ angle in equation 2) against A) Form I and B) Form $1{ }^{1} \mathrm{H}$ and ${ }^{13} \mathrm{C}$ chemical shift $\Delta \delta_{\text {Experimental }}$ data. Conformations differ by $15^{\circ}$ intervals at torsion angle R1 C7 - R1 N8 R2 C1 - R2 C6. Form I and Form II conformations are indicated with dashed orange and magenta lines, respectively.

A) Vs. $\Delta \delta_{\text {Experimental }}$ (ppm) Form I

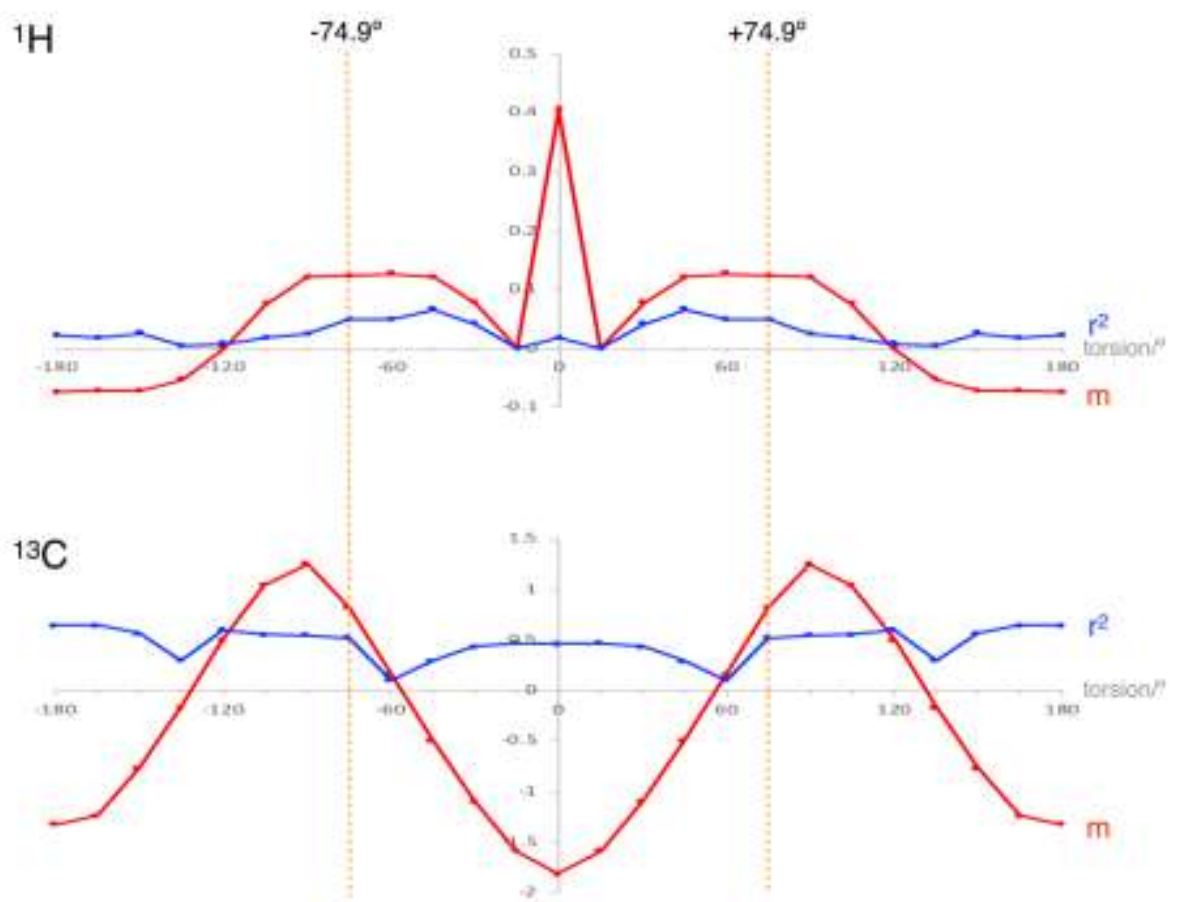

B) $V s . \Delta \delta_{\text {Experimental }}(\mathrm{ppm})$ Form II

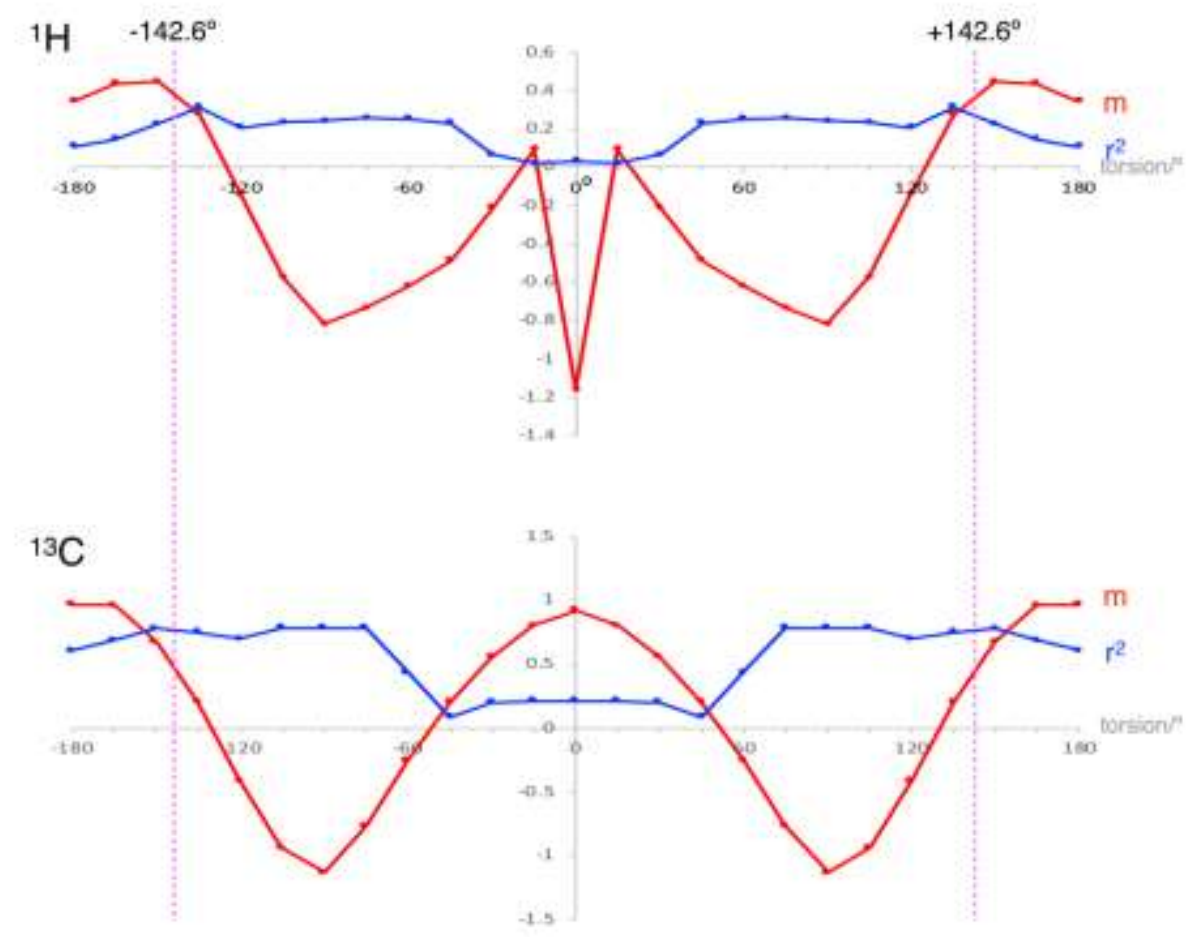


Supplementary Figure S17 - Correlation coefficient $r$ for plots of $\Delta \delta_{\text {Calculated-Isolated conformation at specific } 15^{\circ} \text { angle }}$ (i.e., replacing $\delta_{\text {solid calc }}$ by $\delta_{\text {Isolated conformation at specific } 15^{\circ} \text { angle }}$ for each $15^{\circ}$ angle in equation 2 ) against A) Form I and B) Form II ${ }^{1} \mathrm{H}$ chemical shift $\Delta \delta_{\text {Experimental }}$ data. Conformations differ by $15^{\circ}$ intervals at torsion angle R1 C7 - R1 N8 - R2 C1 - R2 C6. Form I and Form II conformations are indicated with dashed orange and magenta lines, respectively.

\section{A) Vs. $\Delta \delta_{\text {Experimental }}(\mathrm{ppm})$ Form I}

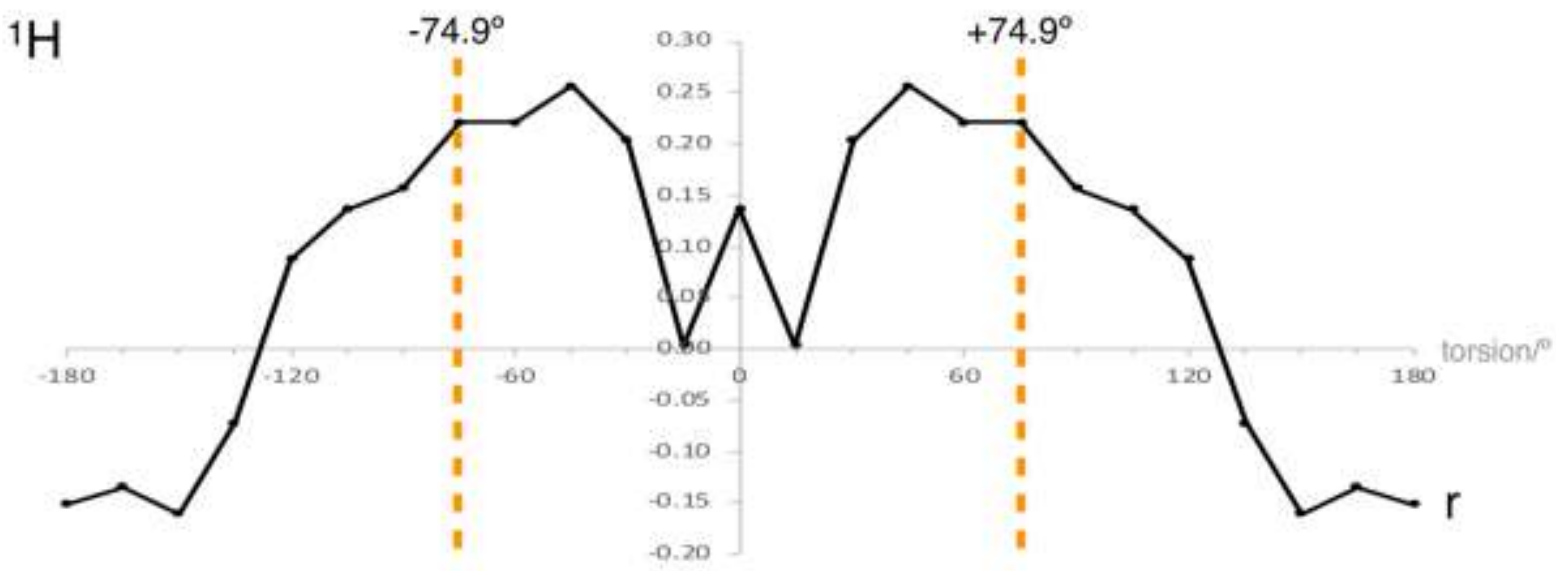

B) Vs. $\Delta \delta_{\text {Experimental }}(\mathrm{ppm})$ Form II

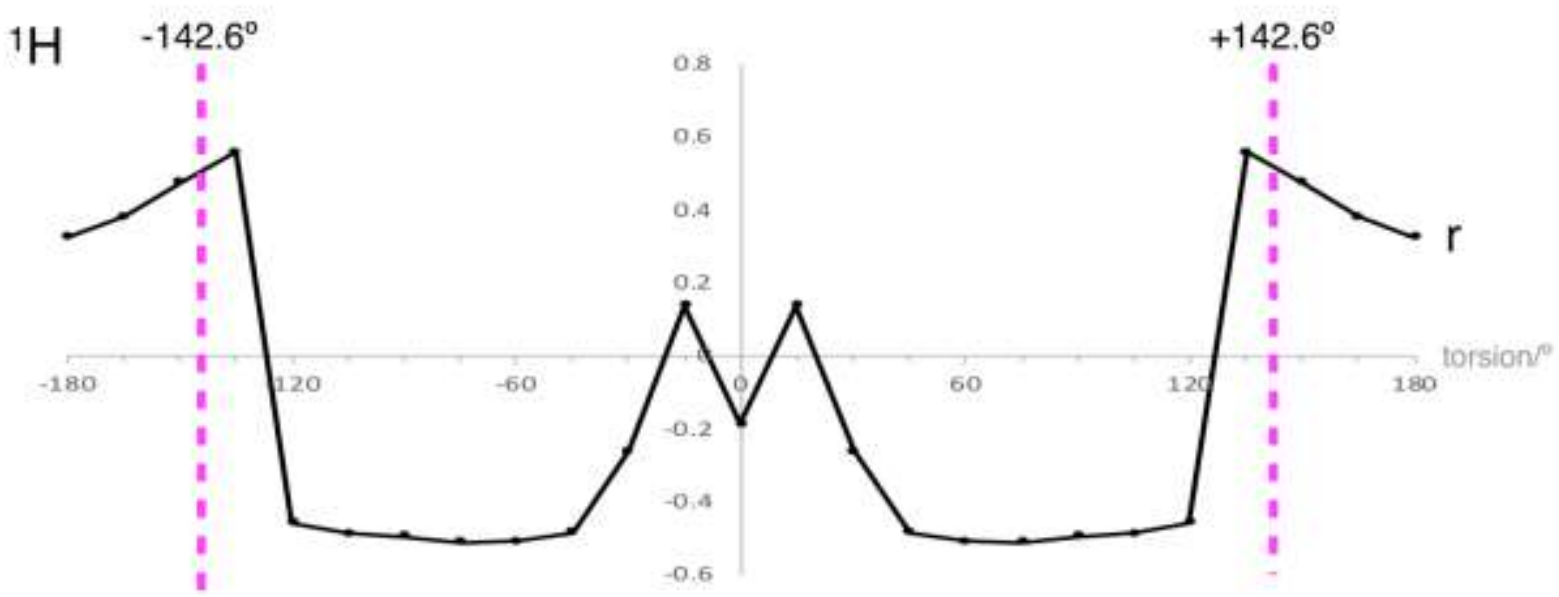




\section{DFT Calculations: Further Details}

Supplementary Figure S18 - Atom numbering (top heavy atoms, bottom hydrogens) and torsion of interest (yellow bonds) according to the CASTEP output (.magres file) shown on an example conformation.
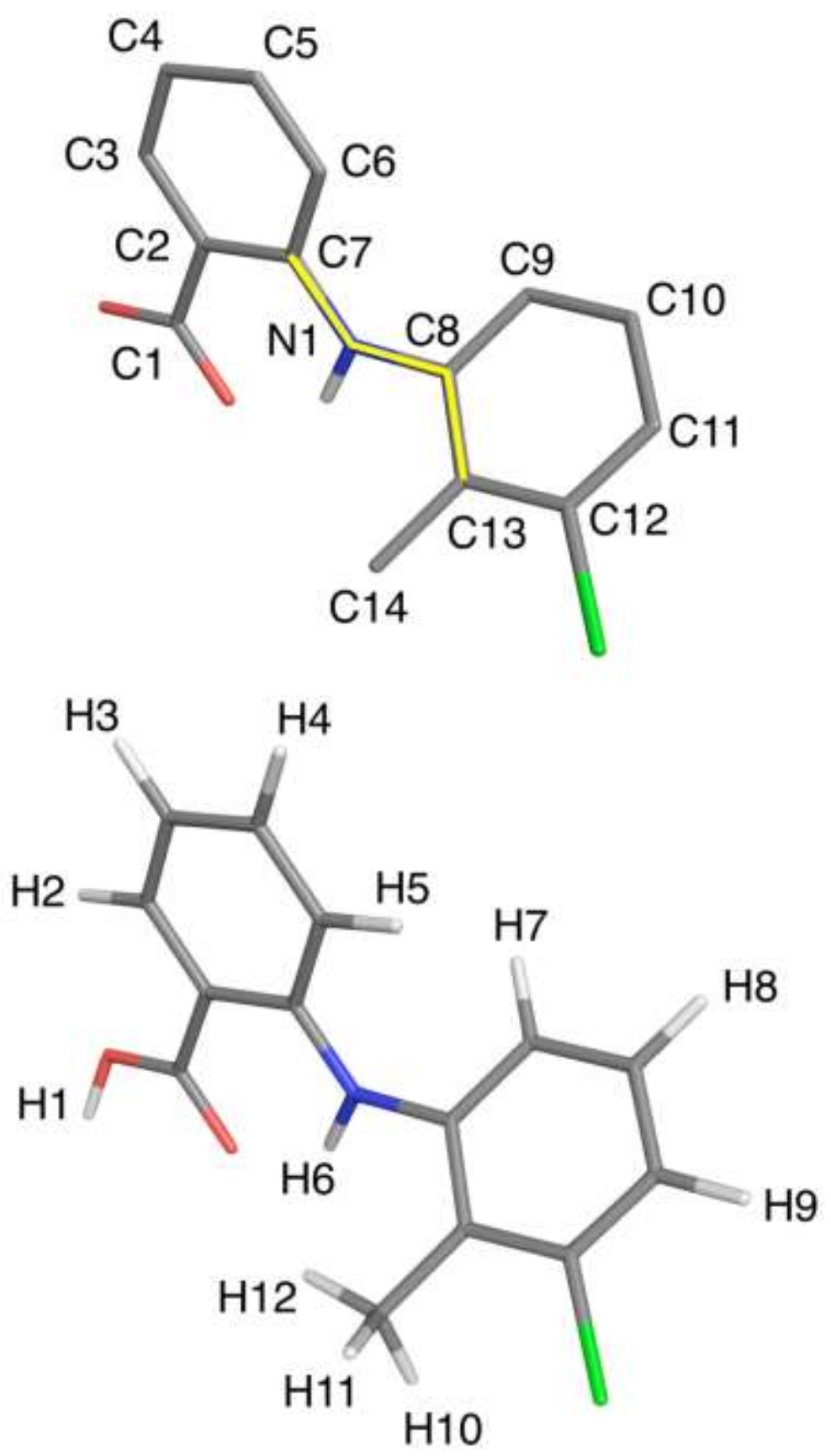

Note that a DFT geometry optimisation on the base conformations (see supplementary Table S4) was performed with fixed torsion angles using the following in the CASTEP .cell file:

\%BLOCK NONLINEAR_CONSTRAINTS
torsion
C $\begin{array}{llllllllll}7 & 0 & 0 & 0 & \mathrm{~N} & 1 & 0 & 0 & 0\end{array}$
C 80000
C 13000

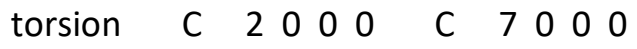
N 10000
C 80000
\%ENDBLOCK NONLINEAR_CONSTRAINTS 


\section{Supplementary Information References}

1. Brown S. P., Prog. Nucl. Magn. Reson. Spectrosc., 2007, 50, 199.

2. Sommer W., Gottwald J., Demco D. E., Spiess H. W., J. Magn. Reson., Ser A., 1995, 113, 131.

3. Schnell I., Lupulescu A., Hafner S., Demco D. E., Spies H. W., J. Magn. Reson., 1998, 133, 61.

4. Gan Z., Amoureux J. P., Trébos, J., Chem. Phys. Lett., 2007, 435, 163.

5. Oas T. G., Griffin R. G., Levitt, M. H., J. Chem. Phys., 1988, 89, 692.

6. Webber A. L., Masiero S., Pieraccini S., Burey J. C., Tatton A. S., luga D., Pham T. N., Spada G. P., Brown S. P., J. Amer. Chem. Soc., 2011, 133, 19777.

7. Costa P. R., Gross J. D., Hong M., Griffin R. G., Chem. Phys. Lett., 1997, 280, 95.

8. Tatton A. S., Bradley J. P., luga, D., Brown, S. P.., Z. Phys. Chemie-Int. J. Res. Phys. Chem. Chem. Phys., 2012, 226, 1187.

9. Hayashi S., Hayamizu K., Bull. Chem. Soc. Jpn., 1991, 64, 688. 